\title{
Gas temperature profiles in galaxy clusters with Swift XRT: observations and capabilities to map near $\boldsymbol{R}_{\mathbf{2 0 0}}$
}

\author{
A. Moretti ${ }^{1}$, F. Gastaldello ${ }^{2}$, S. Ettori ${ }^{3,4}$, and S. Molendi ${ }^{2}$ \\ 1 INAF, Osservatorio Astronomico di Brera, via E. Bianchi 46, 23807 Merate (LC), Italy \\ e-mail: alberto.moretti@brera.inaf.it \\ 2 INAF, via E. Bassini 15, 20133 Milano, Italy \\ 3 INAF, Osservatorio Astronomico di Bologna, via Ranzani 1, 40127 Bologna, Italy \\ ${ }^{4}$ INFN, Sezione di Bologna, viale Berti Pichat 6/2, 40127 Bologna, Italy
}

Received 21 October 2010 / Accepted 21 December 2010

\section{ABSTRACT}

\begin{abstract}
Aims. We investigate the possibility of using the X-ray telescope (XRT) on board the Swift satellite to improve the current accuracy of the intra-cluster medium (ICM) temperature measurements in the region close to the virial radius of nearby clusters.

Methods. We present the spectral analysis of the Swift XRT observations of 6 galaxy clusters and their temperature profiles in the regions within 0.2-0.6 $r_{200}$. Four of them are nearby famous and very well studied objects (Coma, Abell 1795, Abell 2029 and PKS0745-19). The remaining two, SWJ1557+35 (Abell 2141) and SWJ0847+13, at redshift $z=0.16$ and $z=0.36$, were serendipitously observed by Swift-XRT. We accurately quantify the temperature uncertainties, with particular focus on the impact of the background scatter (both instrumental and cosmic). We extrapolate these results and simulate a deep observation of the external region of Abell 1795 which is assumed here as a case study. In particular we calculate the expected uncertainties in the temperature measurement as far as $r_{200}$.

Results. We find that, with a fairly deep observation (300 ks), the Swift XRT would be able to measure the ICM temperature profiles in the external regions as far as the virial radius, significantly improving the best accuracy among the previous measurements. This can be achieved thanks to the unprecedented combination of good PSF over the full field of view and very accurate control of the instrumental background.

Conclusions. Somehow unexpectedly we conclude that, among currently operating telescope, the Swift-XRT is the only potentially able to improve the current accuracy in plasma temperature measurement at the edges of the cluster potential. This will be true until a new generation of low-background and large field of view telescopes, aimed to the study of galaxy clusters, will operate. These observations would be of great importance in developing the observing strategy for such missions.
\end{abstract}

Key words. galaxies: clusters: intracluster medium - X-rays: diffuse background - methods: statistical

\section{Introduction}

Galaxy clusters form by the hierarchical accretion of cosmic matter. They reach the virial equilibrium over a volume that defines the regions where the pristine gas accretes on the dark matter (DM) halo through gravitational collapse and is heated up to millions degrees through adiabatic compression and shocks. The end products of this accretion process exhibit in the X-ray band similar radial profiles of surface brightness (Vikhlinin et al. 1999; Neumann 2005; Ettori \& Balestra 2009), plasma temperature (e.g. Allen et al. 2001; Vikhlinin et al. 2005; Leccardi \& Molendi 2008) and gravitational mass distribution (e.g. Pointecouteau et al. 2005). The measurement of the properties of the ICM have been enormously improved thanks to the arcsec resolution and large collecting area of Chandra and $X M M-N e w t o n$, but still remain possible only where the X-ray emission can be well resolved against the background (both instrumental and cosmic). While the X-ray surface brightness and gas density can be estimated in few cases above $0.7 r_{200}$ (Vikhlinin et al. 1999; Neumann 2005; Ettori \& Balestra 2009, e.g.), the ICM temperature, requiring more than an order of magnitude in net counts than the surface brightness to be firmly measured, can be reasonably well constrained up to a fraction $(\sim 0.5-0.6)$ of the virial radius (e.g. Vikhlinin et al. 2006;
Leccardi \& Molendi 2008; Ettori \& Molendi 2010; Arnaud et al. 2010). The regions not-yet observed are expected to retain most of the information on the processes that characterize the accretion and evolution within the cluster of the main baryonic component (Roncarelli et al. 2006; Rasheed et al. 2010). It is therefore crucial to obtain direct measurements of the cluster properties at these large radii where very important processes for the evolution of the clusters take place. Very recently, Suzaku, thanks to its low background and high sensitivity, has been able to map roughly (i.e. with a spatial resolution limited to $>4^{\prime}$ ) the regions close to the virial radius, providing the first estimate of the gas temperature in 6 objects (Fujita et al. 2008; George et al. 2009; Reiprich et al. 2009; Bautz et al. 2009; Kawaharada et al. 2010; Hoshino et al. 2010). The aim of this paper is to show that the X-ray telescope (XRT) (Burrows et al. 2005) on board the Swift satellite (Gehrels et al. 2004) can improve the accuracy of these measurements with fairly deep observations. To this end, we present the first spectral analysis of the archived Swift observations of nearby clusters (Sect. 2). Since this is a non-standard analysis for the Swift-XRT data and is used and presented here for the first time, we discuss in detail the techniques adopted. In particular in Sect. 3 we describe the procedure we developed to estimate the background and its systematic uncertainty. To calculate how much this uncertainty affects the temperature 

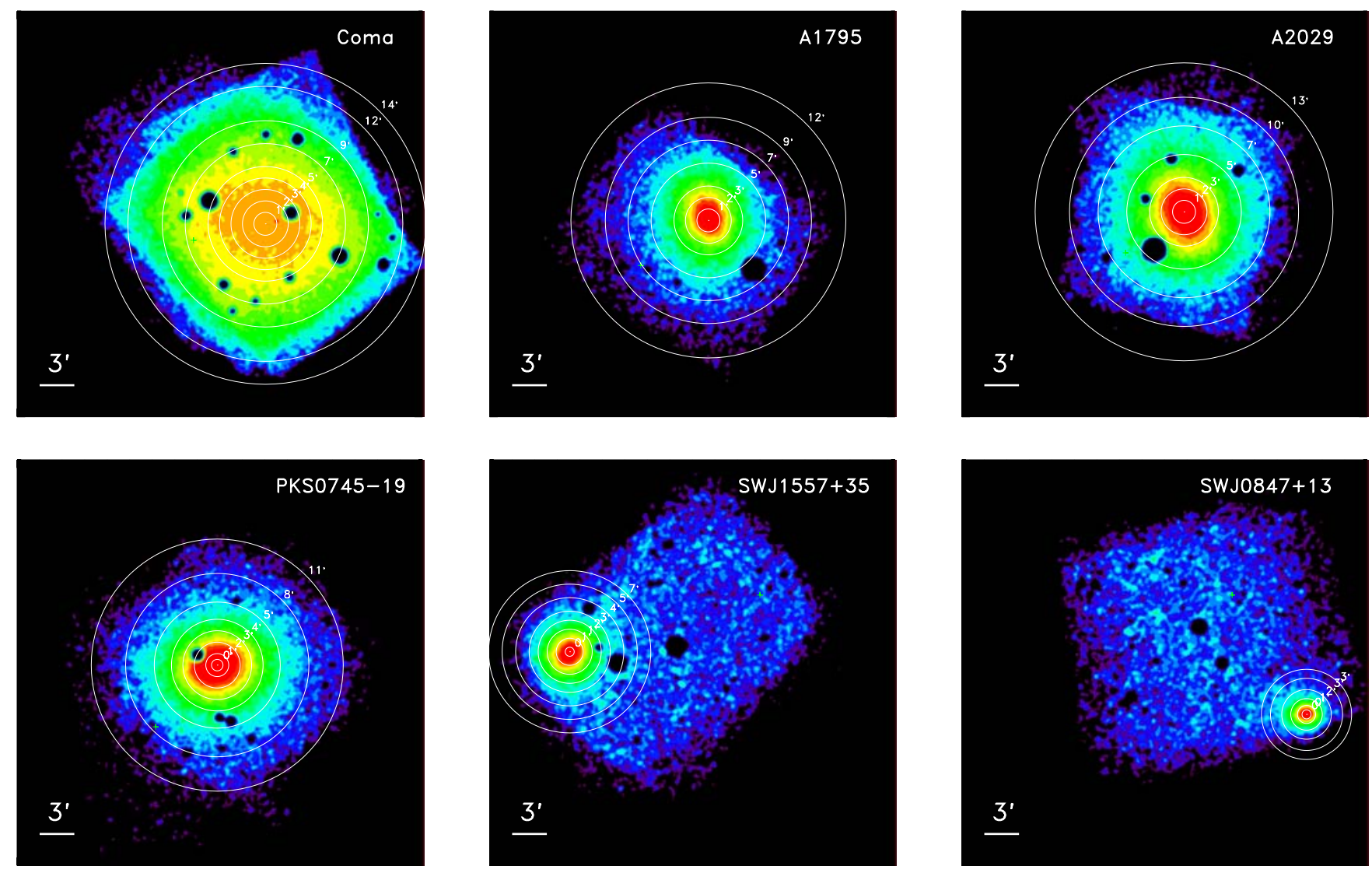

Fig. 1. Images for the six objects in our sample, with detected sources excised. Data are smoothed with a 30" Gaussian filter. Superimposed are the circular extraction regions used for the spectral analysis. Circular regions around the detected sources are excised.

measurement we performed a series of Monte Carlo simulations of thermal

spectrum at different level of surface brightness (described in Sect. 4). Finally in Sect. 5 we used these results to properly and robustly evaluate the expected uncertainties on the gas temperature measurements at $r_{200}{ }^{1}$ on a simulated 300 ks observation of the well studied cluster Abell 1795.

Throughout this paper we assume $H_{0}=70 \mathrm{~km} \mathrm{~s}^{-1}$ and $\Omega_{\lambda}=0.73$ and $\Omega_{\mathrm{m}}=0.27$, which are the default values in the XSPEC(v12.5) software. All errors are quoted at $68 \%$ confidence level for one parameter of interest, unless otherwise specified.

\section{Data reduction and analysis procedures}

\subsection{The sample}

The X-ray telescope (XRT) on board the Swift satellite (Gehrels et al. 2004), uses a Wolter I mirror set, originally designed for the JET-X telescope (Citterio et al. 1994), to focus X-rays $(0.2-10 \mathrm{keV})$ onto a XMM-Newton/EPIC MOS CCD detector (Burrows et al. 2005). The effective area of the telescope $\left(\sim 150 \mathrm{~cm}^{2}\right.$ at $\left.1.5 \mathrm{keV}\right)$ is $\sim 3$. smaller than one single XMM-Newton MOS. The PSF, similar to XMM-Newton, is characterized by an half energy width (HEW) of $\sim 18^{\prime \prime}$ at $1.5 \mathrm{keV}$

\footnotetext{
1 The radius that defines the sphere enclosing a mean cluster density that is 200 times the critical value at the cluster's redshift. Here we use $r_{200}$ and virial radius indifferently and we calculate $r_{200}$ using the scaling relations given by Arnaud et al. (2005) $r_{200}=1714 \frac{(k T / 5)^{0.5}}{h z}[\mathrm{kpc}]$ where $h z=\sqrt{\left((1+z)^{3} \Omega_{\mathrm{m}}+\Omega_{\Lambda}\right)}$.
}

(Moretti et al. 2005). As part of the Swift scientific payload, since the start of the mission (November 2004), Swift-XRT has been mostly used to observe GRB afterglows and other variable sources. Galaxy clusters are observed mostly for calibration purposes or serendipitously.

We cross-correlated the XRT archive updated on April 2010 with the BAX database (http://bax.ast.obs-mip.fr/) searching for cluster observed with more than 20000 events registered in the $0.7-7.0 \mathrm{keV}$ energy band. The sample consists of four famous and very well studied objects (Fig. 1, Table 1). For these clusters the XRT archive data allowed us to measure the projected temperature profile as far as the edge of the telescope field of view corresponding to $0.2-0.6 r_{200}$.

We added 2 more clusters to this sample; these are SWJ1557+3530 and SWJ0847+1331 at redshift $z=0.15$ and $z=0.36$ respectively (according to X-ray spectral analysis, see below), which were serendipitously observed in the field of the follow-up observations of GRB090409 and GRB051016B and are the two highest signal objects of the SXCS catalog (Moretti et al. 2007; Moretti et al. in prep.). They are already cataloged as Abell $2141(z=0.1579)$ and as WHL J084749.3+133140 (Wen et al. 2009) $(z=0.36)$ respectively. They are X-ray bright $\left(L_{\mathrm{X}} \sim 3\right.$ and $7 \times 10^{44} \mathrm{erg} \mathrm{s}^{-} 1$ in the $0.5-2.0 \mathrm{keV}$ rest frame energy band) with average temperatures of 6.0 and $6.8 \mathrm{keV}$ respectively. They can be considered rich also form the point of view of the optical classification having 35 and 42 galaxies associated according the MaxBCG (Koester et al. 2007) and the WHL catalogs respectively. The cataloged redshift measurements are in very good agreement with ours (see Table 1). Differently from 
Table 1. Cluster sample.

\begin{tabular}{|c|c|c|c|c|c|c|c|c|}
\hline Name & $\begin{array}{c}\text { RA, Dec } \\
\text { [deg] }\end{array}$ & $z$ & $\begin{array}{c}k T \\
{[\mathrm{keV}]}\end{array}$ & References & $\begin{array}{c}r_{200} \\
{\left[{ }^{\prime}, \mathrm{Mpc}\right]}\end{array}$ & $\begin{array}{c}r_{\max } \\
{\left[r_{200}\right]}\end{array}$ & $\begin{array}{l}\text { Exp. } \\
{[\mathrm{ks}]}\end{array}$ & $\begin{array}{c}N_{\mathrm{H}} \\
10^{20} \mathrm{~cm}^{-2}\end{array}$ \\
\hline Coma & $194.9392+27.9429$ & 0.023 & $8.25 \pm 0.01$ & Arnaud et al. (2001) & $78.2[2.18]$ & 0.17 & 43.7 & 0.80 \\
\hline Abe & $207.2183+26.5903$ & 0.062 & $6.12 \pm 0.05$ & Vikhlinin et al. (2006) & $25.7[1.84]$ & 0.41 & 20.3 & 1.32 \\
\hline Abell 2029 & $227.7336+5.74440$ & 0.077 & $8.47 \pm 0.09$ & Vikhlinin et al. (2006) & $26.7[2.16]$ & 0.43 & 42.3 & 3.25 \\
\hline PKS0745-19 & $116.8799-19.2948$ & 0.102 & $7.97 \pm 0.28$ & Arnaud et al. (2005) & $18.1[2.06]$ & 0.58 & 63.1 & 41.8 \\
\hline SWJ1557+3530 & $239.4287+35.5073$ & 0.158 & $6.79 \pm 0.25$ & Owen et al. (1995) & 11.5 [1.89] & 0.62 & 180.1 & 0.20 \\
\hline SWJ0847+1331 & $131.9550+13.5278$ & $0.358 \pm 0.005$ & $6.02 \pm 0.34$ & this work & $5.3[1.56]$ & 0.50 & 203.1 & 3.23 \\
\hline
\end{tabular}

the first four, these two objects do not entirely fill the field of view allowing us to perform a consistency check of our background model through a comparison with the local background (see below).

In Table 1 the Mean spectroscopic temperatures of the first four clusters are from the references reported in the fifth column; the remaining 2 are calculated from our data (see text). $r_{200}$ are estimated by adopting the scaling relations (Arnaud et al. 2005) and here are expressed in units of arcmin and Mpc. $r_{\max }$ is the center of the most external annulus for which we measured the temperature, here expressed in units of $r_{200}$.

\subsection{Data reduction}

Data reduction was performed using the standard software (HEADAS software, v6.8, CALDB version 20091130, Nov. 2009) and following the procedures described in the instrument user guide ${ }^{2}$. At variance with these, we excluded the external (Detx $>90$ and Detx $<510$ ) CCD columns which are affected by the presence of out-of-time-events from corner calibration sources (see Moretti et al. 2009, for a detailed map of the CCD and a discussion on the XRT background). This left us with a nominal field of view of $16.5^{\prime} \times 18.9^{\prime}\left(0.087 \mathrm{deg}^{2}\right)$. Different observations of the same objects and relative exposure maps were merged by means of the the extractor and farith tasks of the HEADAS software respectively.

Before performing the spectral analysis, we ran the CIAO wavedetect and eliminated all the events within the circles centered on the detected source positions with radius such that the PSF surface brightness equals the background (typically $\lesssim 10$ pixels $\lesssim 23^{\prime \prime}$ ). Vignetted and non-vignetted exposure maps were built consequently accounting for the excised regions.

\subsection{Spectral analysis}

We measured the temperature profiles, binning the data in projected annuli of amplitude $1.0^{\prime}-2.5^{\prime}$ moving from central to the external part of the image (Fig. 1). The statistics of the extracted spectra strongly vary going from the center, where the extraction region size is determined only by the PSF toward the outskirts of the sources, where the size of the regions is determined by ensuring a minimum of 200 source counts in the $0.7-2.0 \mathrm{keV}$ energy band. Because the XRT standard software does not include any specific task for the spectral analysis of the extended sources, we developed a procedure made by combination of ad-hoc IDL routines and Ftools scripts to calculate the vignetting and the exposure corrections and to estimate the background.

For the vignetting calculation, we, first, created a vignetting map at $1.5 \mathrm{keV}$, dividing the exposure map by the un-vignetted

\footnotetext{
2 http://heasarc.nasa.gov/docs/swift/analysis/ documentation
}

exposure. Then, we created the maps at different energies, using the vignetting analytical description given by Moretti et al. (2004). Finally we used the exposure and vignetting maps weighted by the cluster surface brightness profile to calculate the effective time and the vignetting correction, for each annulus. As the vignetting depends on energy, in order to take into account for these corrections in the spectral analysis we opportunely modified the nominal ARF file.

The evaluation of the background is surely the most important and delicate step in the procedure. This is primarily because the virial regions $\left(R_{200}>15^{\prime}\right)$ of nearby massive clusters subtend the entire field of view. Therefore, the background cannot be estimated locally. For our purposes we consider the total XRT background as the sum of an instrument (NXB) and a cosmic (XRB) component. The latter one is, in turn, the sum of a galactic (GXRB) and extragalactic (CXRB) component, with very different spectral and spatial characteristics.

We evaluated the instrumental background (NXB) using the data collected, during the observations, in the regions of the detector which are not exposed to the sky (NESR, Not-ExposedSky-Regions). These are four different small regions (2507 pixels each) close to the CCD boundary and delimited by the field of view and the corner sources (Moretti et al. 2009).

To estimate the XRB we used a statistical approach: we made use of a large number (135) of deep GRB follow-up observations to study the XRB characteristics. Because GRB are uniformly distributed, these are deep exposures on random positions of the sky, totally uncorrelated with already known bright X-ray sources, and provide us with a data set which is very well suited to statistically characterize the XRB. In the following we will refer to this data set as the blank fields (BFs). A similar data-set was used by Moretti et al. (2009) to perform an absolute measurement of the CXRB in the 1.5-7 keV range. Here, we found that all the BF spectra, in the $0.7-7.0 \mathrm{keV}$ energy band can be well described by the sum of a bremsstrahlung and an absorbed power law, representing the GXRB and CXRB respectively.

We defer the detailed description of the instrumental and cosmic background analysis together with the full evaluation of the systematic errors in temperature measurements to the next two sections (Sects. 3, 4), while here we focus on the remaining details of the analysis and the results.

As high energy limit we use $7.0 \mathrm{keV}$, because, between 7 and $10 \mathrm{keV}$, the instrumental background is significantly higher due to the presence of Nickel and Gold lines. We set the low energy limit at $0.7 \mathrm{keV}$ both because around $0.5 \mathrm{keV}$ response matrix calibrations are more problematic due to the presence of uncorrected charge traps (Godet et al. 2009), and because we found that, below $0.7 \mathrm{keV}$, the XRB is less reproducible due to the presence of some extra local components (see also Kuntz \& Snowden 2000). In the case of Coma, both brightness and temperature are so high that we easily extended our analysis up to $10.0 \mathrm{keV}$. In the case of PKS0745-19, which is on the Galactic 
plane, we used the $1.0-7.0 \mathrm{keV}$ band to reduce the uncertainty on the background estimate which is higher at lower Galactic latitudes.

We used XSPEC(v12.5) Cash statistics to fit the cluster+XRB spectra from the annular regions with a bremss+wabs $\times$ (pow+apec) model, where the absorbed APEC model represents the cluster emission, using the NESR spectra as background. We used literature red-shift values (http://bax.ast.obs-mip.fr/) for all the clusters but for SWJ0847+1331 which is not yet cataloged. In this case we measured the redshift using the $\mathrm{Fe}-\mathrm{K}$ line in the $\mathrm{X}$-ray spectrum from the central $1^{\prime}$ radius. We left the metallicity parameter free to vary only when the estimated counts of the sources exceed 5000 , freezing it to the $0.3 Z_{\odot}$ in the other cases. Concerning the Galactic absorption, for each cluster, we took advantage of the good statistics in the three central annuli, and we let the $N_{\mathrm{H}}$ absorbing column vary in the fit. As we found, in all the cases, a $<2 \sigma$ consistency of the best fit value with the value derived from the HI Galaxy map (Kalberla et al. 2005), we froze the Galactic $N_{\mathrm{H}}$ absorbing columns to these values. In three cases these estimate coincide with the XMM best fit values (Coma, Abell 1795, PKS0745-19 differences <3\%) (Snowden et al. 2008), while, in the case of Abell 2029, the difference is $20 \%$.

We tried to fit the cluster data together with XRB, but the statistics of the data did not allow us to constrain the XRB parameters together with the cluster ones, even when the XRB is a significant fraction of the total signal. Instead, for all our spectra, we froze the four XRB parameters to the median values of the BF distributions, just re-normalizing them by the area of the extraction region. We calculated the systematic uncertainties of this approach reproducing the same procedure on a large sample of simulated spectra (Sect. 4). Finally we calculated the total error as the quadratic sum of the statistical error (the one provided by XSPEC) and the systematic one. The results are shown in the Fig. 2 and reported in details in Table 2. In this table, for each annulus, we report the radii, the area, the effective exposure time accounting for exposure maps and vignetting, the mean cluster surface brightness, the total events, the source/total ratio and the best fits values. The statistical error contribution to the total temperature errors are reported in parenthesis.

We note that in the case of the PKS0745-19 the systematic errors are probably underestimated, due to the fact that this cluster lies on the Galactic plane, whereas our XRB statistical analysis is suitable for the extragalactic sky (Galactic latitude $>20^{\circ}$ ). We mitigated this problem limiting the spectral analysis for this particular cluster to energies higher than $1.0 \mathrm{keV}$.

In Fig. 3 we plot the XRT temperature measurements of the first four clusters, compared with XMM-Newton results from Snowden et al. (2008): we generally found a good agreement between the two instruments without any appreciable bias or systematic trend as shown by the linear fit. On the other hand, calculating the scatter as the variance of the (XRT)-(XMM-Newton) difference distribution, divided by the errors, we measured a scatter which is slightly larger than the expected: $1.32 \pm 0.17$ instead of 1.0. Most of this scatter can be ascribed to the outer regions of the cluster PKS0745-19, for which, as said, the errors are underestimated. Indeed excluding them lowers the scatter to $1.20 \pm 0.18$, consistent with the expectation at $1.1 \sigma$ level. We obtained the same result $(1.22 \pm 0.20)$ artificially increasing the PKS0745-19 errors by a factor 1.4. In this comparison we performed the spectral analysis exactly in the same regions used by (Snowden et al. 2008), while data plotted in Fig. 2 and listed in Table 2 are in (slightly) different regions, modified in order to optimize XRT data statistics.
Due to small apparent size and the good statistics SWJ1557+3530 and SWJ0847+1331 are the best cases, in the XRT archive, to perform a consistency check between temperature measurement performed with a locally evaluated and a background model: results are in good agreement, as shown in the last two panels of Fig. 2.

\section{Background analysis}

To study both the NXB and XRB we made use of a large sample of blank fields (BFs). We selected the 135 (31 at low Galactic latitude) GRB follow up observations from January 2006 to April 2009 with a nominal exposure time longer than $10 \mathrm{ks}$ and shorter than $300 \mathrm{ks}$ (Fig. 4). We reduced these data and eliminated detected sources, following the same procedure we used for cluster data and described in Sect. 2.3.

\subsection{Instrumental background}

In the Swift-XRT CCD, we have the opportunity of estimating the NXB directly from the signal registered in the NESR during the observations. This method allows to optimally map the NXB time variations which occur due to changes in the satellite environment at any time scales from minutes to years and that are very difficult to be accounted for otherwise. Indeed, as shown in Fig. 5, the raw spectrum registered in the NESR regions, once re-normalized for the area, accurately reproduces the signal registered in whole the field of view beyond $7 \mathrm{keV}$, where the instrument background completely dominates the BF signal. On the contrary NESR are in the CCD corners and do not map the NXB spatial variations within the detector. Moretti et al. (2009), using the data collected during the unique (and unrepeatable) observation performed with the camera shutter closed (September 2007), found a linear gradient in the NXB signal along the vertical direction of the CCD, with the bottom regions being $20 \%$ fainter than the top regions. This gradient was found achromatic within the statistical errors $(1 \sigma \sim 5 \%)$. Here, we used all the BFs stacked data (6.3 Ms in total) to study the NXB spatial pattern. First, comparing the 4 NESR spectra we found that while the upper regions (NESR 1, 2) and lower (NESR 3, 4) are consistent between each other both in intensity and spectral shape, the upper ones are significantly different from the lower ones (Fig. 5). Then, to study the NXB spatial pattern we used the unresolved signal from all the BFs stacked data in the 7.0-10. keV energy band. As said, in absence of bright sources, in this range, the signal is almost purely instrumental, dominated by the Nickel and Gold fluorescence lines (Fig. 5). From these data we confirmed that the NXB has a linear vertical gradient with slope very well consistent with the one found by Moretti et al. (2009). The scatter in the stacked BF 7-10. keV image measured in $50 \times 50$ pixel cells is $\sim 8 \%$, (1.5\% statistical); correcting the image by the linear gradient reduces this scatter to $\sim 2 \%$ (Fig. 6). If we assume that the NXB linear gradient is achromatic this means that, starting from the NESR signal, we can recover the NXB with $\sim 2.3 \%$ accuracy ( $1 \sigma$, plus the statistical uncertainty) in any position of the detector. The assumption of achromaticity is justified by the analysis results of the shutter-closed observation.

\subsection{Cosmic background}

In order to study the statistical properties of the cosmic components of the background we performed the spectral analysis of the 135 BFs unresolved signal, using the NESR spectrum 

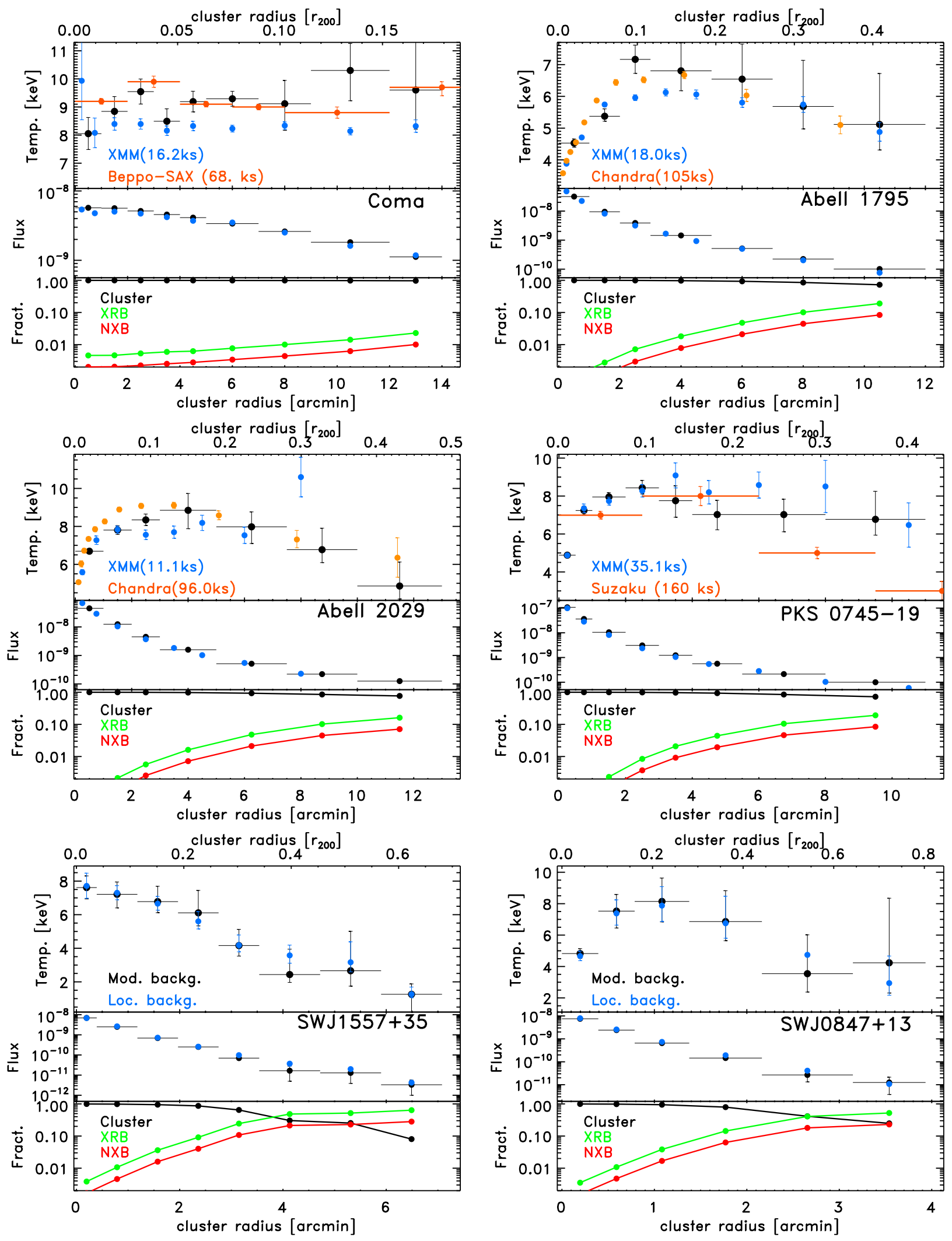

Fig. 2. Upper panels: the projected temperature profiles of the six clusters of our sample. For the first four objects, Swift XRT measurements (black points) are compared with XMM-Newton (blue points) Snowden et al. (2008). In the Coma plot we also report the BeppoSAX numbers from De Grandi \& Molendi (2002). In the Abell 2029 and Abell 1795 plots we report the Chandra measurements from Vikhlinin et al. (2006). In the PKS0745-19 we report the Suzaku points (George et al. 2009). In the case of SWJ1557+35 and SWJ0847+13 we compare measurements obtained with locally evaluated and modeled background. Middle panels: fluxes in the $0.3-10 . \mathrm{keV}$ band in $\mathrm{cgs}^{-2} \mathrm{deg}^{-2}$ units compared with XMM-Newton values from Snowden et al. (2008), in the first four cases, and with the value after local background subtraction in the remaining two. Lower panels: for each extraction region we plot the relative value of the $0.5-2.0 \mathrm{keV}$ band flux for the cluster (black), XRB (green) and NXB (red). 
Table 2. Spectral analysis results.

\begin{tabular}{|c|c|c|c|c|c|c|c|c|}
\hline Cluster & $\begin{array}{c}\text { Ann. rad. } \\
{\left[{ }^{\prime}\right]}\end{array}$ & $\begin{array}{c}\text { Area } \\
{\left[\mathrm{deg}^{2}\right]}\end{array}$ & $\begin{array}{l}\text { Exp. } \\
{[\mathrm{ks}]}\end{array}$ & $\begin{array}{c}S B_{[0.5-2 .]} \\
{\left[\mathrm{erg} \mathrm{s}^{-1} \mathrm{~cm}^{-2} \mathrm{deg}^{-2}\right]}\end{array}$ & Tot. evt. $[0.7-2]$. & $\begin{array}{c}S /(S+B) \\
{[\%]}\end{array}$ & $\begin{array}{c}k T \\
{[\mathrm{keV}]}\end{array}$ & $\begin{array}{l}Z \\
Z_{\odot} \\
\end{array}$ \\
\hline Coma & $0.0-1.0$ & 8.7E-04 & 41.9 & $5.72 \mathrm{E}-09 \pm 3.04 \mathrm{E}-10$ & $3.1 \mathrm{e}+03$ & 99.5 & $8.1_{-0.6(0.6)}^{+0.6(0.6)}$ & $0.30_{0.00}^{+0.00}$ \\
\hline Coma & $1.0-2.0$ & $2.5 \mathrm{E}-03$ & 41.6 & $5.64 \mathrm{E}-09 \pm 2.89 \mathrm{E}-10$ & $8.6 e+03$ & 99.4 & $8.8_{-0.5(0.5)}^{+0.5(0.5)}$ & $0.24_{-0.08}^{+0.08}$ \\
\hline Coma & $2.0-3.0$ & $4.0 \mathrm{E}-03$ & 41.8 & $5.14 \mathrm{E}-09 \pm 2.61 \mathrm{E}-10$ & $1.2 \mathrm{e}+04$ & 99.4 & $9.5_{-0.4(0.4)}^{+0.4(0.4)}$ & $0.22_{-0.07}^{+0.07}$ \\
\hline Coma & $3.0-4.0$ & $6.1 \mathrm{E}-03$ & 42.0 & $4.57 \mathrm{E}-09 \pm 2.31 \mathrm{E}-10$ & $1.7 \mathrm{e}+04$ & 99.3 & $8.5_{-0.3(0.3)}^{+0.4(4)}$ & $0.20_{-0.05}^{+0.05}$ \\
\hline Coma & $4.0-5.0$ & $7.5 \mathrm{E}-03$ & 41.8 & $4.13 \mathrm{E}-09 \pm 2.09 \mathrm{E}-10$ & $1.9 \mathrm{e}+04$ & 99.2 & $9.2_{-0.4(0.4)}^{+0.4(0.4)}$ & $0.25_{-0.05}^{+0.06}$ \\
\hline Coma & $5.0-7.0$ & $1.9 \mathrm{E}-02$ & 40.5 & $3.38 \mathrm{E}-09 \pm 1.70 \mathrm{E}-10$ & $3.8 \mathrm{e}+04$ & 99.0 & $9.3_{-0.3(0.3)}^{+0.3(3)}$ & $0.28_{-0.04}^{+0.04}$ \\
\hline Coma & $7.0-9.0$ & $2.6 \mathrm{E}-02$ & 32.3 & $2.61 \mathrm{E}-09 \pm 1.31 \mathrm{E}-10$ & $3.3 e+04$ & 98.8 & $9.1_{-0.7(0.3)}^{+0.8(0.3)}$ & $0.19_{-0.04}^{+0.05}$ \\
\hline Coma & $9.0-12.0$ & 5.3E-02 & 16.5 & $1.82 \mathrm{E}-09 \pm 9.20 \mathrm{E}-11$ & $2.2 \mathrm{e}+04$ & 98.2 & $10.3_{-0.8(0.4)}^{+1.0 .4)}$ & $0.45_{-0.08}^{+0.08}$ \\
\hline Coma & $12.0-14.0$ & $2.9 \mathrm{E}-02$ & 5.6 & $1.12 \mathrm{E}-09 \pm 6.03 \mathrm{E}-11$ & $2.7 \mathrm{e}+03$ & 97.1 & $9.6_{-1.2(1.0)}^{+1.7(1.5)}$ & $0.30_{0.00}^{+0.00}$ \\
\hline Abell2029 & $0.0-1.0$ & 8.7E-04 & 38.7 & $4.49 \mathrm{E}-08 \pm 2.26 \mathrm{E}-09$ & $2.4 \mathrm{e}+04$ & 99.9 & $6.7_{-0.1(0.1)}^{+0.1(0.1)}$ & $0.58_{-0.05}^{+0.05}$ \\
\hline Abell2029 & $1.0-2.0$ & 2.6E-03 & 39.4 & $1.25 \mathrm{E}-08 \pm 6.29 \mathrm{E}-10$ & $2.0 \mathrm{e}+04$ & 99.8 & $7.8_{-0.2(0.2)}^{+0.2(0.1)}$ & $0.36_{-0.05}^{+0.05}$ \\
\hline Abell2029 & $2.0-3.0$ & 4.4E-03 & 40.6 & $4.50 \mathrm{E}-09 \pm 2.29 \mathrm{E}-10$ & $1.2 \mathrm{e}+04$ & 99.3 & $8.3_{-0.3(0.3)}^{+0.3(0.3)}$ & $0.43_{-0.06}^{+0.07}$ \\
\hline Abell2029 & $3.0-5.0$ & $1.2 \mathrm{E}-02$ & 41.2 & $1.59 \mathrm{E}-09 \pm 8.07 \mathrm{E}-11$ & $1.2 \mathrm{e}+04$ & 98.1 & $8.8_{-0.7(0.4)}^{+0.9(0.5)}$ & $0.27_{-0.06}^{+0.07}$ \\
\hline Abell2029 & $5.0-7.5$ & 2.7E-02 & 40.0 & $5.12 \mathrm{E}-10 \pm 2.62 \mathrm{E}-11$ & $8.9 e+03$ & 94.4 & $8.0_{-0.7(0.4)}^{+0.8(0.4)}$ & $0.22_{-0.07}^{+0.08}$ \\
\hline Abell2029 & $7.5-10.0$ & $3.8 \mathrm{E}-02$ & 28.9 & $2.21 \mathrm{E}-10 \pm 1.17 \mathrm{E}-11$ & $4.4 \mathrm{e}+03$ & 88.5 & $6.8_{-0.6(0.4)}^{+1.0(0.8)}$ & $0.30_{0.00}^{+0.00}$ \\
\hline Abell2029 & $10.0-13.0$ & 4.3E-02 & 9.6 & $1.26 \mathrm{E}-10 \pm 7.66 \mathrm{E}-12$ & $1.2 \mathrm{e}+03$ & 83.5 & $4.9_{-0.7(0.6)}^{+0.9(0.8)}$ & $0.30_{0.00}^{+0.00}$ \\
\hline Abell1795 & $0.0-1.0$ & 8.7E-04 & 20.0 & $3.16 \mathrm{E}-08 \pm 1.61 \mathrm{E}-09$ & $1.0 \mathrm{e}+04$ & 99.9 & $4.5_{-0.1(0.1)}^{+0.1(0.1)}$ & $0.46_{-0.06}^{+0.07}$ \\
\hline Abell1795 & $1.0-2.0$ & $2.6 \mathrm{E}-03$ & 19.8 & $9.50 \mathrm{E}-09 \pm 4.86 \mathrm{E}-10$ & $8.7 \mathrm{e}+03$ & 99.7 & $5.4_{-0.2(0.2)}^{+0.2(0.2)}$ & $0.36_{-0.06}^{+0.07}$ \\
\hline Abell1795 & $2.0-3.0$ & 4.4E-03 & 19.2 & $3.90 \mathrm{E}-09 \pm 2.02 \mathrm{E}-10$ & $5.2 \mathrm{e}+03$ & 99.3 & $7.2_{-0.4(0.4)}^{+0.4(0.4)}$ & $0.30_{0.00}^{+0.00}$ \\
\hline Abell1795 & $3.0-5.0$ & $1.4 \mathrm{E}-02$ & 19.2 & $1.45 \mathrm{E}-09 \pm 7.48 \mathrm{E}-11$ & $6.4 \mathrm{e}+03$ & 98.0 & $6.8_{-0.5(0.3)}^{+0.7(0.4)}$ & $0.30_{0.00}^{+0.00}$ \\
\hline Abell1795 & $5.0-7.0$ & $1.9 \mathrm{E}-02$ & 17.8 & $5.16 \mathrm{E}-10 \pm 2.76 \mathrm{E}-11$ & $3.1 \mathrm{e}+03$ & 94.8 & $6.5_{-0.6(0.5)}^{+0.8(0.6)}$ & $0.30_{0.00}^{+0.00}$ \\
\hline Abell1795 & $7.0-9.0$ & 2.7E-02 & 15.9 & $2.23 \mathrm{E}-10 \pm 1.26 \mathrm{E}-11$ & $1.9 \mathrm{e}+03$ & 89.3 & $5.7_{-0.6(0.6)}^{+0.7(0.7)}$ & $0.30_{0.00}^{+0.00}$ \\
\hline Abell1795 & $9.0-12.0$ & 4.0E-02 & 11.4 & $1.01 \mathrm{E}-10 \pm 6.41 \mathrm{E}-12$ & $1.0 \mathrm{e}+03$ & 79.9 & $5.1_{-0.8(0.7)}^{+1.2(1.1)}$ & $0.30_{0.00}^{+0.00}$ \\
\hline PKS0745-19 & $0.0-0.5$ & 2.2E-04 & 75.6 & $1.08 \mathrm{E}-07 \pm 5.42 \mathrm{E}-09$ & $3.3 e+04$ & 100.0 & $4.9_{-0.1(0.1)}^{+0.1(0.1)}$ & $0.44_{-0.04}^{+0.04}$ \\
\hline PKS0745-19 & $0.5-1.0$ & $6.5 \mathrm{E}-04$ & 77.2 & $3.62 \mathrm{E}-08 \pm 1.82 \mathrm{E}-09$ & $2.9 \mathrm{e}+04$ & 99.9 & $7.2_{-0.2(0.2)}^{+0.2(0.2)}$ & $0.37_{-0.04}^{+0.04}$ \\
\hline PKS0745-19 & $1.0-2.0$ & $2.4 \mathrm{E}-03$ & 77.1 & $1.05 \mathrm{E}-08 \pm 5.28 \mathrm{E}-10$ & $3.0 \mathrm{e}+04$ & 99.7 & $8.0_{-0.2(0.2)}^{+0.2(0.2)}$ & $0.38_{-0.04}^{+0.04}$ \\
\hline PKS0745-19 & $2.0-3.0$ & 4.1E-03 & 77.8 & $3.09 \mathrm{E}-09 \pm 1.57 \mathrm{E}-10$ & $1.5 \mathrm{e}+04$ & 99.0 & $8.4_{-0.3(0.3)}^{+0.4(0.4)}$ & $0.43_{-0.06}^{+0.06}$ \\
\hline PKS0745-19 & $3.0-4.0$ & $6.1 \mathrm{E}-03$ & 77.9 & $1.23 \mathrm{E}-09 \pm 6.28 \mathrm{E}-11$ & $9.4 \mathrm{e}+03$ & 97.6 & $7.8_{-0.7(0.4)}^{+0.8(0.4)}$ & $0.30_{0.00}^{+0.00}$ \\
\hline PKS0745-19 & $4.0-5.5$ & $1.2 \mathrm{E}-02$ & 74.9 & $5.61 \mathrm{E}-10 \pm 2.88 \mathrm{E}-11$ & $8.5 e+03$ & 95.1 & $7.0_{-0.6(0.4)}^{+0.8(0.5)}$ & $0.30_{0.00}^{+0.00}$ \\
\hline PKS0745-19 & $5.5-8.0$ & $2.9 \mathrm{E}-02$ & 68.8 & $2.14 \mathrm{E}-10 \pm 1.10 \mathrm{E}-11$ & $7.8 \mathrm{e}+03$ & 88.1 & $7.0_{-0.8(0.6)}^{+0.8(0.6)}$ & $0.30_{0.00}^{+0.00}$ \\
\hline PKS0745-19 & $8.0-11.0$ & 4.9E-02 & 40.9 & $9.97 \mathrm{E}-11 \pm 5.37 \mathrm{E}-12$ & $4.2 \mathrm{e}+03$ & 77.8 & $6.8_{-0.9(0.6)}^{+1.5(1.2)}$ & $0.30_{0.00}^{+0.00}$ \\
\hline SWJ1557+35 & $0.0-0.4$ & $1.3 \mathrm{E}-04$ & 187.3 & $6.91 \mathrm{E}-09 \pm 3.70 \mathrm{E}-10$ & $2.7 \mathrm{e}+03$ & 99.6 & $7.6_{-0.7(0.7)}^{+0.7(0.7)}$ & $0.30_{0.00}^{+0.00}$ \\
\hline SWJ1557+35 & $0.4-1.2$ & $1.1 \mathrm{E}-03$ & 176.3 & $2.49 \mathrm{E}-09 \pm 1.28 \mathrm{E}-10$ & $7.8 \mathrm{e}+03$ & 98.9 & $7.2_{-0.6(0.4)}^{+0.7(0.4)}$ & $0.30_{0.00}^{+0.00}$ \\
\hline SWJ1557+35 & $1.2-2.0$ & $2.2 \mathrm{E}-03$ & 170.1 & $6.90 \mathrm{E}-10 \pm 3.62 \mathrm{E}-11$ & $4.4 e+03$ & 96.1 & $6.8_{-0.6(0.4)}^{+0.7(0.4)}$ & $0.30_{0.00}^{+0.00}$ \\
\hline SWJ1557+35 & $2.0-2.8$ & $3.1 \mathrm{E}-03$ & 163.3 & $2.51 \mathrm{E}-10 \pm 1.37 \mathrm{E}-11$ & $2.5 e+03$ & 90.4 & $6.1_{-0.7(0.6)}^{+0.8(0.6)}$ & $0.30_{0.00}^{+0.00}$ \\
\hline SWJ1557+35 & $2.8-3.5$ & 4.3E-03 & 154.5 & $7.00 \mathrm{E}-11 \pm 4.35 \mathrm{E}-12$ & $1.3 e+03$ & 75.9 & $4.2_{-0.6(0.4)}^{+0.9(0.8)}$ & $0.30_{0.00}^{+0.00}$ \\
\hline SWJ1557+35 & $3.5-4.7$ & 7.6E-03 & 123.5 & $1.66 \mathrm{E}-11 \pm 1.40 \mathrm{E}-12$ & $8.7 \mathrm{e}+02$ & 49.7 & $2.4_{-0.4(0.3)}^{+0.7(0.5)}$ & $0.30_{0.00}^{+0.00}$ \\
\hline SWJ1557+35 & $4.7-5.9$ & $1.0 \mathrm{E}-02$ & 101.0 & $1.29 \mathrm{E}-11 \pm 1.23 \mathrm{E}-12$ & $8.5 e+02$ & 42.4 & $2.7_{-0.6(0.5)}^{+1.1(0.6)}$ & $0.30_{0.00}^{+0.00}$ \\
\hline SWJ1557+35 & $5.9-7.1$ & $1.3 \mathrm{E}-02$ & 87.6 & $3.32 \mathrm{E}-12 \pm 6.12 \mathrm{E}-13$ & $6.9 \mathrm{e}+02$ & 21.6 & $1.3_{-0.3(0.2)}^{+1.2(0.5)}$ & $0.30_{0.00}^{+0.00}$ \\
\hline SWJ0847+13 & $0.0-0.4$ & $1.3 \mathrm{E}-04$ & 104.9 & $7.50 \mathrm{E}-09 \pm 4.08 \mathrm{E}-10$ & $2.2 \mathrm{e}+03$ & 99.7 & $4.8_{-0.3(0.3)}^{+0.3(0.3)}$ & $0.30_{0.00}^{+0.00}$ \\
\hline SWJ0847+13 & $0.4-0.8$ & $4.0 \mathrm{E}-04$ & 100.9 & $2.41 \mathrm{E}-09 \pm 1.34 \mathrm{E}-10$ & $1.7 \mathrm{e}+03$ & 98.9 & $\begin{array}{r}-0.3(0.8) \\
7.5_{-0.9(0.8)}^{+1.1(0.8)}\end{array}$ & $0.30_{0.00}^{+0.00}$ \\
\hline SWJ0847+13 & $0.8-1.4$ & $1.1 \mathrm{E}-03$ & 94.7 & $6.53 \mathrm{E}-10 \pm 3.82 \mathrm{E}-11$ & $1.2 \mathrm{e}+03$ & 95.9 & $8.1_{-1.2(1.0)}^{+1.5(1.3)}$ & $0.30_{0.00}^{+0.00}$ \\
\hline SWJ0847+13 & $1.4-2.2$ & $2.4 \mathrm{E}-03$ & 84.4 & $1.46 \mathrm{E}-10 \pm 1.00 \mathrm{E}-11$ & $6.2 e+02$ & 84.8 & $6.9_{-1.2(1.1)}^{+2.0(1.8)}$ & $0.30_{0.00}^{+0.00}$ \\
\hline SWJ0847+13 & $2.2-3.1$ & 4.6E-03 & 73.2 & $2.67 \mathrm{E}-11 \pm 2.71 \mathrm{E}-12$ & $3.7 \mathrm{e}+02$ & 58.5 & $3.5_{-1.0(0.9)}^{+1.8(1.6)}$ & $0.30_{0.00}^{+0.00}$ \\
\hline SWJ0847+13 & $3.1-3.9$ & $4.0 \mathrm{E}-03$ & 85.9 & $1.26 \mathrm{E}-11 \pm 2.19 \mathrm{E}-12$ & $2.5 e+02$ & 37.6 & $4.2_{-1.8(1.5)}^{+3.7(3.2)}$ & $0.30_{0.00}^{+0.00}$ \\
\hline
\end{tabular}

as instrumental background. Freezing the value of the Galactic absorbing Hydrogen column, for each field, to Kalberla et al. (2005) values, we found that the unresolved emission in the $0.7-7.0 \mathrm{keV}$ can always be well modeled by a thermal component (bremsstrahlung) plus an absorbed power law, accounting for GXRB and CXRB respectively.

From Snowden et al. (1998) and Kuntz \& Snowden (2000) we know that a physically motivated emission model of the XRB 


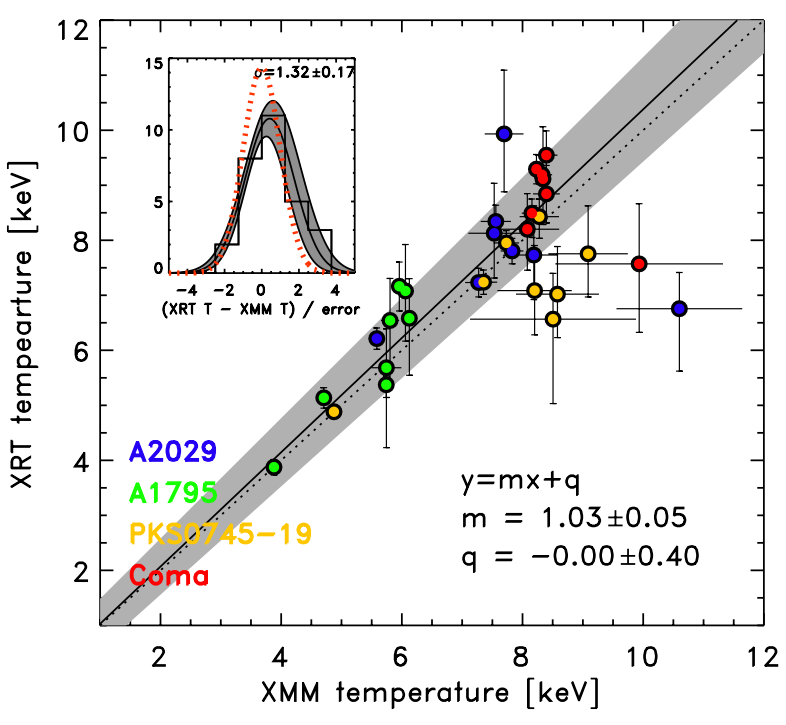

Fig. 3. Swift-XRT and XMM-Newton temperature measurement comparison. The continuous line is the linear fit to the data, while the dotted line is the expected distribution. In the inset the scatter of the measurement is shown with the thick black line. The best fit with its uncertainties is shown with thin black lines and grey area, while the expected scatter is drawn with red dotted line.

below $1 \mathrm{keV}$ should consist of, at least, two components, the first of Galactic origin, the second from local hot bubble. To avoid this complexity in the modeling of the data, we limited our analysis to energies higher than $0.7 \mathrm{keV}$. Although not completely physically motivated, using a simple Bremsstrahlung provided us an accurate phenomenological description of the data in this energy range with a limited number of parameters. This is suitable to our goal, whereas a physical description of the galactic thermal emission is beyond the scope of the present work (and the quality of our data at low energies).

Indeed we found good $\chi^{2}$ values for all the 135 fields (upper panel of Fig. 7). Splitting the sample in low $(<20)$ and high latitude Galactic fields we found that fitting the latter always yielded $\chi^{2}<1.3$, while the formers present slightly larger scatter. The soft thermal component typically contributes to $\sim 50 \%$ of the total emission $\lesssim 1 \mathrm{keV}$, while it is negligible beyond $1.5 \mathrm{keV}$. On the other hand, as we will see in the next Section, neglecting it, could significantly affect the temperature measurement in a regime where the cluster emission is comparable. As expected, we found that high and low latitude fields have different statistical properties, the latter sample presenting a larger scatter in the parameter distributions. As the cluster in the present sample are all (but PKS0745-19) observed at high Galactic latitude we restricted our analysis to the extra Galactic fields. The parameter space of the best fit values of the 104 high Galactic latitude BFs is shown in Fig. 8. We used these data in two ways. First, from these distributions we derived the median spectrum (lower panel of Fig. 7, Fig. 8 and Table 3) used as XRB background component in the cluster spectral analysis (see Sect. 2.3). Then, we used these models as input templates for the simulations employed to quantify the systematic temperature uncertainties (Sect. 4).

Approximately $\sim 20 \%$ of the unresolved emission, in the XRT images, is due to stray light contamination from XRB sources outside the field of view (Moretti et al. 2009). This contamination does not significantly affect our procedures, but it should be taken into account to compare the normalization of
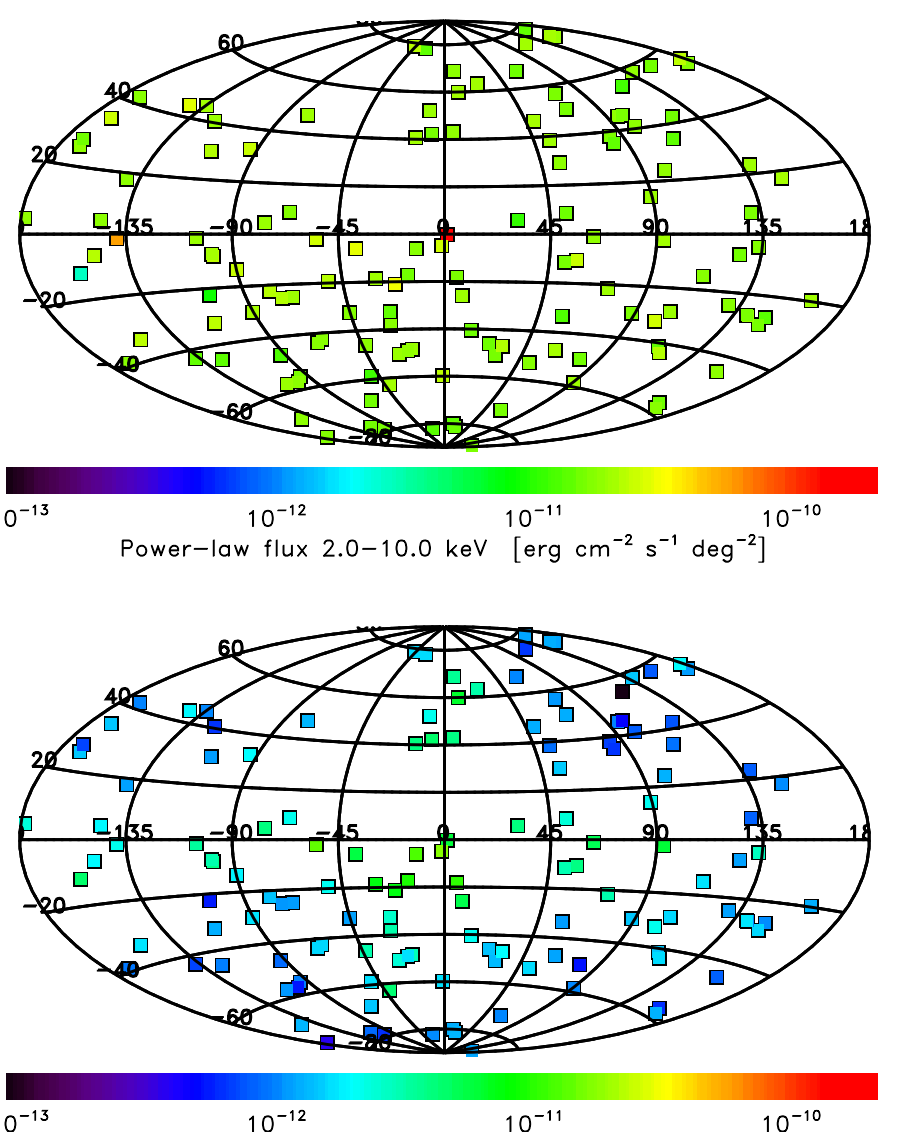

Fig. 4. The Aitoff projection of the Galactic coordinates of the non resolved emission in the blank fields (BFs). Upper panel: the CXRB flux in the 2.0-10.0 keV band. Lower panel: the GXRB flux in the $0.5-2.0 \mathrm{keV}$ band.

our BF spectra with the absolute measurements of the CXRB and GXRB.

\subsection{Time variability}

Time variations of both cosmic and instrument background level are observed in the X-ray telescopes. In the Swift XRT particle flux variations in the satellite environment affecting the NXB, are monitored and accounted for by the NESR data (see Sect. 3.1). More insidious are the variations due to the solar wind exchange (SWCX) producing a diffuse photon emission at $\mathrm{C}, \mathrm{O}$, $\mathrm{Mg}, \mathrm{Ne}$ energies with different timescales from seconds to days. We quantified the impact of SWCX on Swift XRT observations studying the light curves in different energy bands both for the cluster data and for the BFs.

We studied the light curve in the soft band $(0.7-2.0 \mathrm{keV})$ and the color curve $(0.7-2.0 / 0.7-7.0 \mathrm{keV})$ of the source free regions of the 2 serendipitous cluster observations (SWJ1557+35 and SWJ0847+13). We excluded the sources as we did for BFs and binned the data in $\sim 1500 \mathrm{~s}$ time intervals ${ }^{3}$. These choices ensured the necessary statistics to study the flux and spectral variations, as the signal registered in a $\mathrm{BF}$ is $\sim 0.1$ count per second in the $0.7-7.0 \mathrm{keV}$ energy band over the whole detector. Results relative to SWJ1557+35 are shown in Fig. 9. The total exposure time of the observation is $200 \mathrm{ks}$ which is split in 149 segments. We considered only the 115 intervals with a duration longer

\footnotetext{
${ }^{3}$ Due to the low orbit of the satellite, the Swift XRT observations are composed by segments of $\sim 1500 \mathrm{~s}$ for each orbit (5700 s).
} 


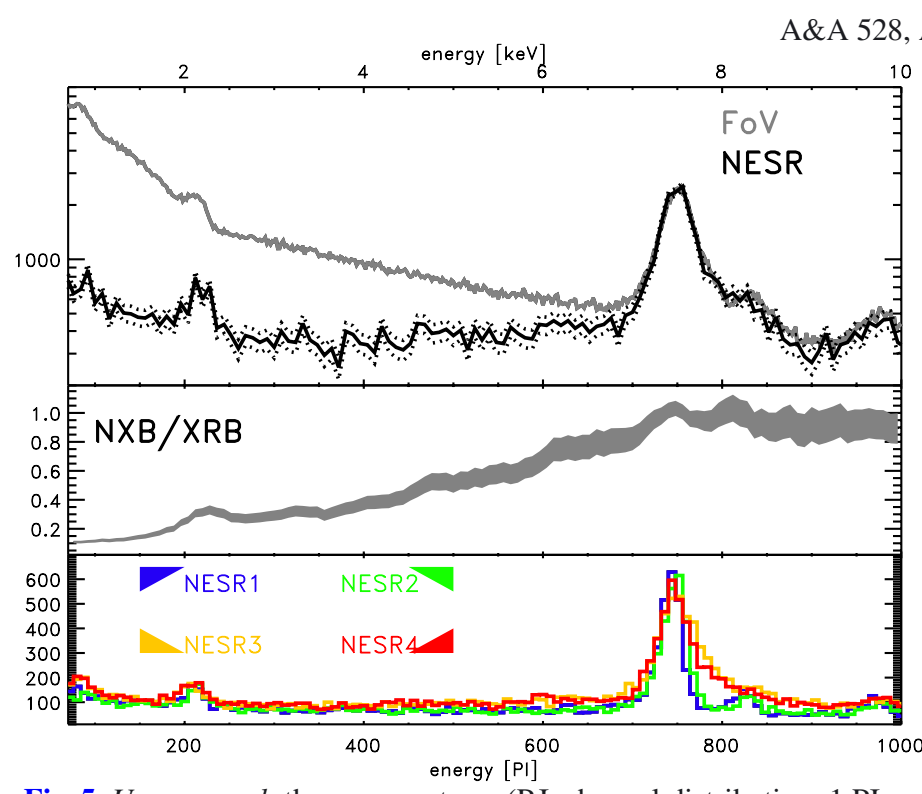

Fig. 5. Upper panel: the raw spectrum (P.I. channel distribution; 1 PI $10 \mathrm{eV}$ ) of a large number of blank fields (BF, in grey) compared with the spectrum registered in the NESR regions in black. Middle panel: the ratio NESR/BF gives the relative importance of the NXB as function of energy in absence of bright sources. Bottom panel: the 4 NESR spectra show not negligible differences.
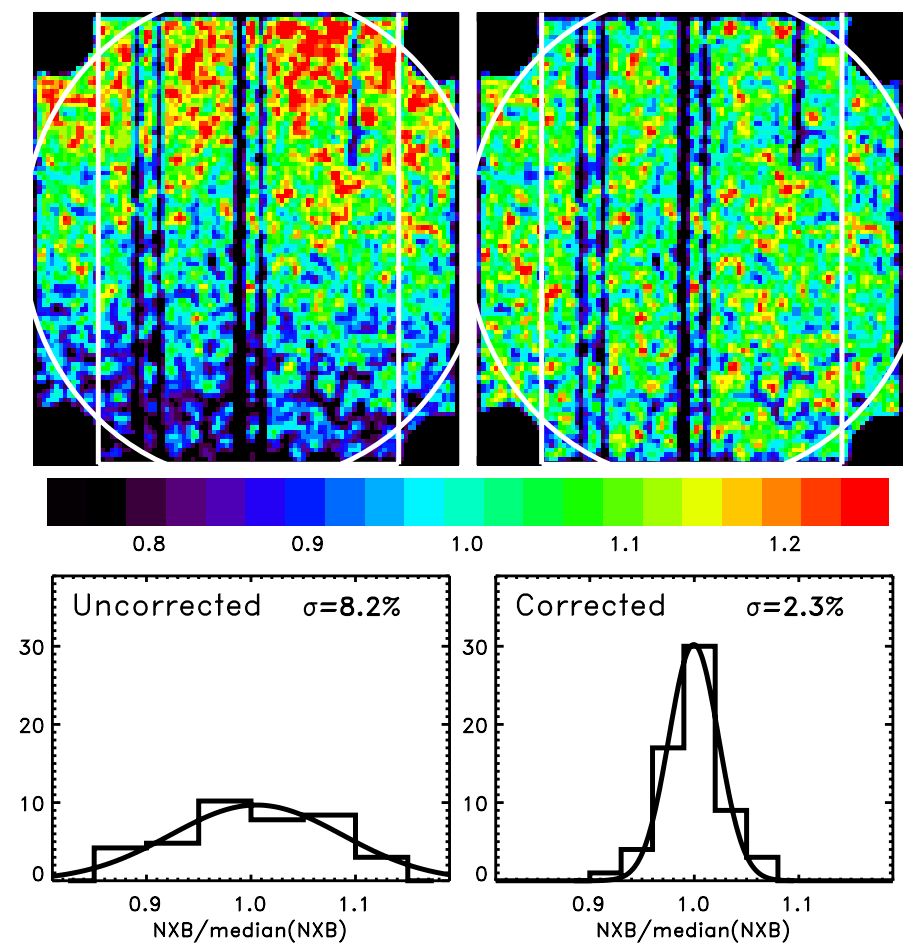

Fig. 6. Upper-left: the CCD map in the $7.0-10.0 \mathrm{keV}$ energy band from $6.3 \mathrm{Ms}$ of BFs, normalized to the central raw to show the NXB vertical gradient; Lower-left: the residual distribution calculated in $50 \times 50$ pixel cells. Upper-right: the CCD map in the 7.0-10.0 keV energy band from $6.3 \mathrm{Ms}$ of BFs, normalized to the central raw to show the NXB vertical gradient, corrected by the linear approximation. Lower-left: the residual distribution calculated in $50 \times 50$ pixel cells with the linear gradient correction applied.

than 1000 s covering more than the $90 \%$ of the total duration $(185 \mathrm{ks})$. We found that both the flux and the color scatter are well consistent with the statistical one. In other words, in this data set, we did not find any detectable signature of SWCX. For SWJ0847+13 we found similar results, while for the four remaining objects the results of this analysis are not conclusive,
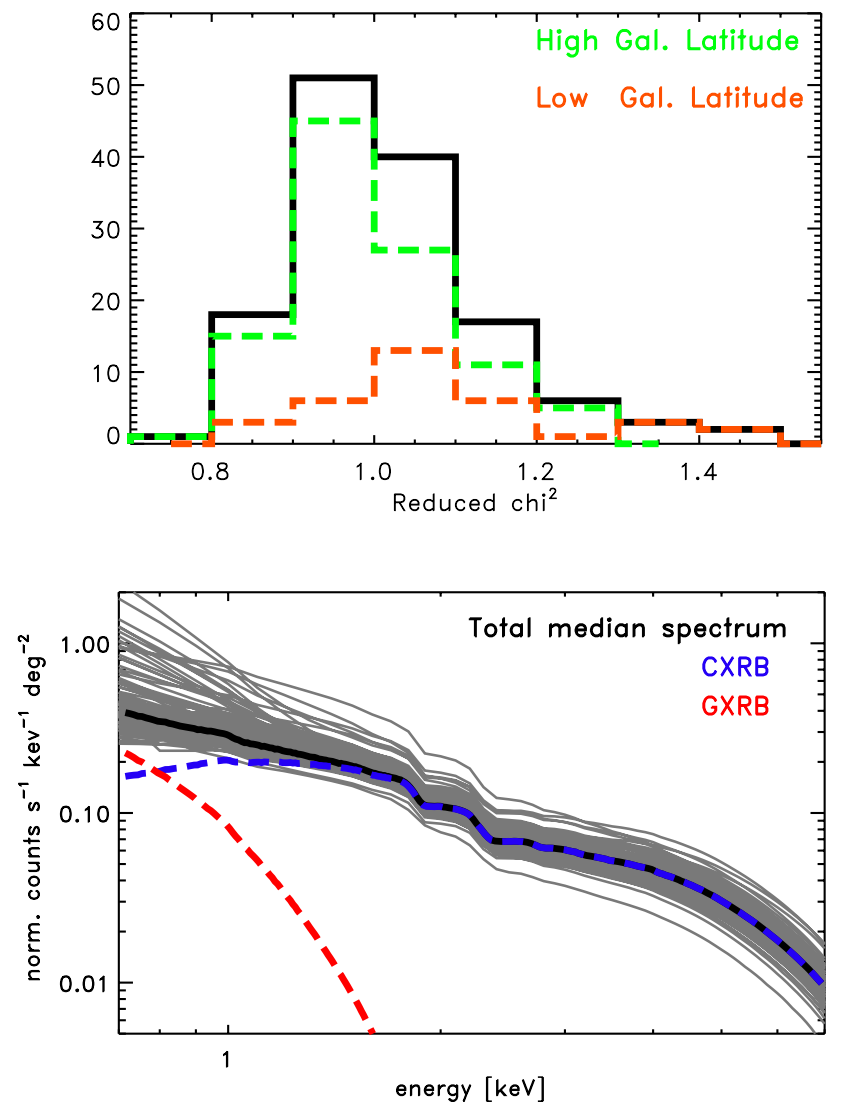

Fig. 7. Upper panel: the reduced $\chi^{2}$ distribution of the 135 fits, here split in high $\left(>20^{\circ}\right)$ and low Galactic latitude fields. Lower panel: the 104 XRB models (grey lines) with the median spectrum in evidence (thick black line). For this we over plot the two components: the Galactic bremsstrahlung (red dashed) and the extragalactic power-law (blue dashed).

due to the very high level of the cluster signal over the entire field of view.

We extended this kind of analysis to all the BFs. To get rid of the cosmic variance, we normalized each observation to its median value and compared the residuals. We found that only $\sim 1.5 \%$ of all the time intervals considered can be identified outliers Fig. 10. Given these findings, we decided to neglect the effect of the SWCX in both our spectral analysis and in our simulations.

\section{Systematic uncertainties in temperature measurement}

In order to evaluate the systematics in the temperature measurement we used XSPEC(v12.5) to simulate a set of cluster+XRB bremss+wabs $\times($ pow + apec $)$ spectra, as observed by the Swift XRT.

To simulate the XRB we used BFs, but we had to consider two further complications. First, CXRB normalization and spectral slope are expected to vary according the image flux limit: the spectrum of the unresolved CXRB emission in deeper images is expected to be fainter and harder (Moretti et al. 2003). Second, the CXRB variance depends on the size of the extraction regions (Kushino et al. 2002; Revnivtsev et al. 2008; Moretti et al. 2009). To account for these effects we split the 

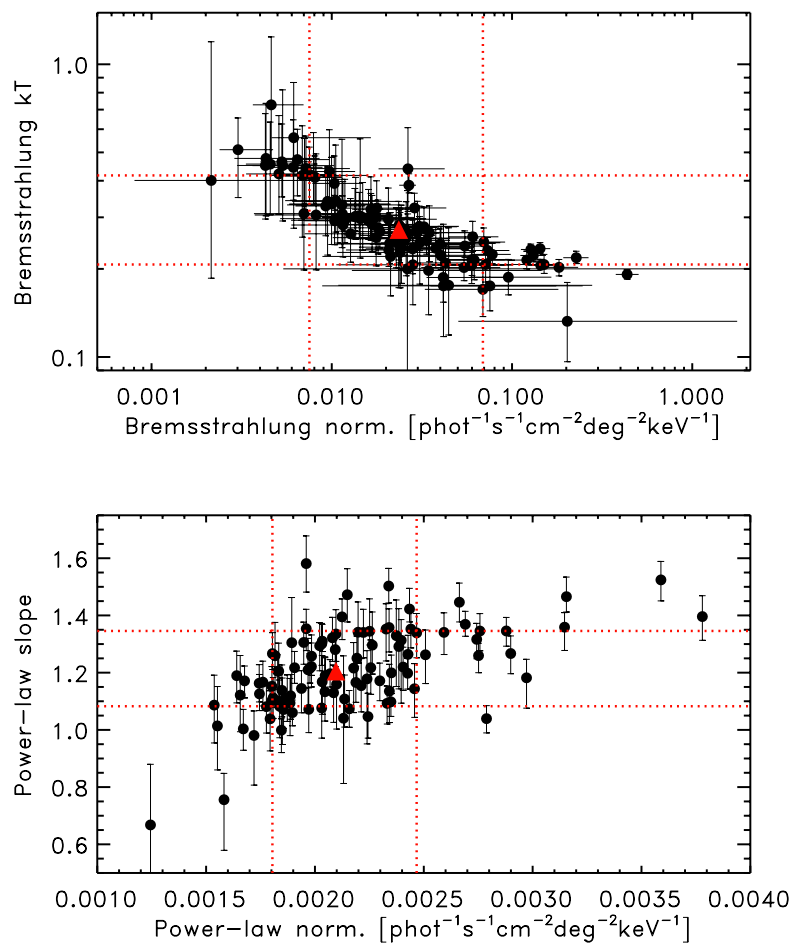

Fig. 8. Upper panel: the bremsstrahlung parameter space of the 104 high Galactic latitude fields. The red dotted lines represent the 16th and 84th percentile of the distributions and the red triangle the median. The red triangle represent the median values adopted as XRB model (Table 3). Lower panel: the power-law parameter space, with same notations.
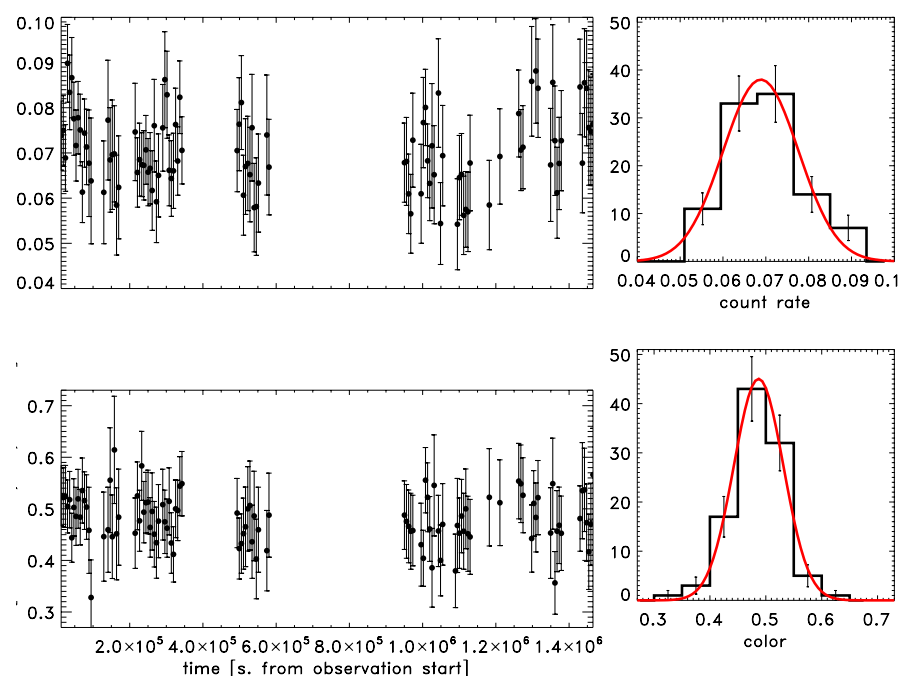

Fig. 9. The light (upper-left panel) and color (lower-left) curve of the unresolved signal in the SWJ1557+35 observations. In the right panels we plot the corresponding distribution together with the Gaussian fit. The observed scatter can be completely ascribed to the statistical error of the measurements.

BF sample in five exposure time bins (10-30, 30-50, 50-75, $75-125,125-200 \mathrm{ks})$ and for each BF we extracted the XRB spectrum from regions of different sizes $(1.0,0.5,0.25$ and 0.1 times the entire field of view).

To simulate the cluster emission we used 37 different cluster temperatures (in the range $1-10 \mathrm{keV}$, step $0.25 \mathrm{keV}$ ) with the
Table 3. The median of the BF spectrum parameter distribution together with the 16th and 84th percentile values.

\begin{tabular}{|c|c|c|c|}
\hline & Median & 16th\% & 84th\% \\
\hline B. norm [phot s $\left.{ }^{-1} \mathrm{~cm}^{-2} \mathrm{deg}^{-2} \mathrm{kev}^{-1}\right]$ & 0.023 & 0.0075 & 0.069 \\
\hline B. $k T[\mathrm{keV}]$ & 0.27 & 0.21 & 0.42 \\
\hline P.L. norm [phot s${ }^{-1} \mathrm{~cm}^{-2} \mathrm{deg}^{-2} \mathrm{kev}^{-1}$ ] & 0.0021 & 0.0018 & 0.0024 \\
\hline P.L. slope [] & 1.20 & 1.08 & 1.34 \\
\hline
\end{tabular}

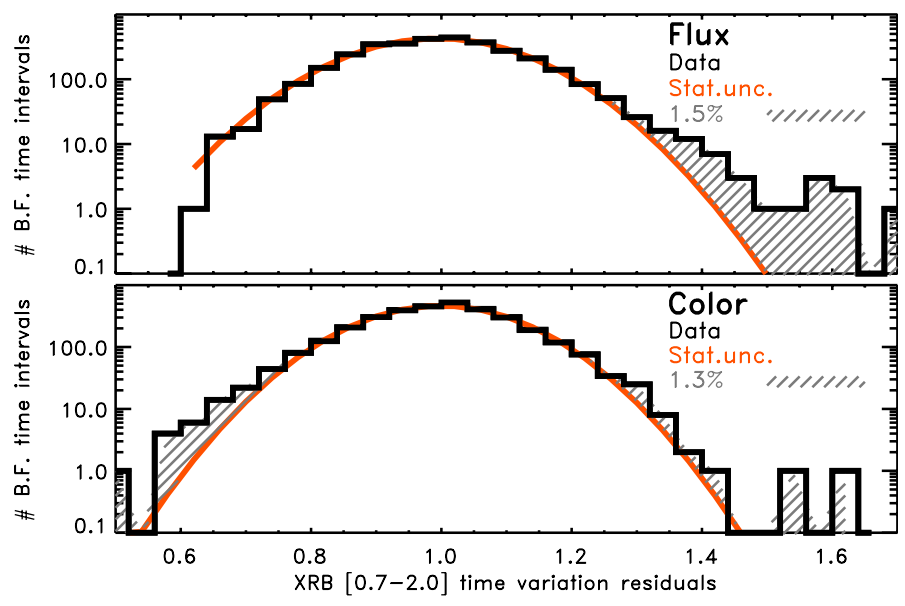

Fig. 10. The residual distribution of the flux and color XRB curve of a collection of BFs. We put in evidence the expected Gaussian distribution from statistical uncertainties and the departures from this. We found that only $\sim 1.5 \%$ of all the time intervals considered can be considered outliers.

same flux $\left(8 \times 10^{-13} \mathrm{erg} \mathrm{s}^{-1} \mathrm{~cm}^{-2}\right)$ and exposure time (100 ks) in order to collect a number of source photons to make the statistical errors negligible (>10000 source counts). Then, for each spectrum we modified the source/background ratio, varying the BACKSCAL keyword, in order to simulate different values of surface brightnesses: 10 steps between $2 \times 10^{-12}$ and $8 \times 10^{-11} \mathrm{erg} \mathrm{s}^{-1} \mathrm{~cm}^{-2} \mathrm{deg}^{-2}$ ). For each step of this $37 \times 10$ grid we simulated 200 different realizations letting the metallicity and $N_{\mathrm{H}}$ normally and randomly varying around the mean values of $0.3 Z_{\odot}$ and $3 \times 10^{20} \mathrm{~cm}^{-2}$ respectively with a scatter of $0.06(20 \%)$ and $6 \times 10^{19}(20 \%)$. Thus, for each simulated cluster we summed an XRB model randomly choosing one of the BF model cycling over the exposures $\times$ region size grid: this resulted in a total of 200 spectra $\times(37 \times 10)$ clusters $(\times 5 \times 4)$ XRB grid. Finally, to each simulation, we added the instrumental background stacking a collection of the NESR data, randomly re-normalizing it by a Gaussian deviation of $2.3 \%$ to account for the uncertainties in the reproduction of the spatial pattern (Sect. 3.1). We used all the BF observed in 2009 , for a total of $\sim 1.5 \mathrm{Ms}$, in order to maximize the statistics and, at the same time, to avoid particle background variation on $\sim 1$ year time scale, as observed in Moretti et al. (2009), which are probably due to variation in the Solar activity.

We fit the simulated data using the same procedure we used for our real data as described in Sect. 2.3. We used a bremss+wabs $\times($ pow + apec $)$ model, freezing the XRB parameters to the BF XRB median spectrum (Sect. 3.2), just normalized for the input area. Moreover, we froze the $N_{\mathrm{H}}$ and metallicity to the mean values $\left(0.3 Z_{\odot}\right.$ and $3 \times 10^{20} \mathrm{~cm}^{-2}$ respectively) and we used the GRB 090618 follow-up observation NXB as background. 
As an example of the simulation outputs, in Fig. 11, we plot the distribution of the results of the fit on the 200 realizations of APEC spectra with different input temperatures $T_{\mathrm{i}}$, for two different values of surface brightness (high surface brightness in the higher panel, low surface brightness in the middle panel). In this case the background was extracted from BF with exposure time within $75-125 \mathrm{ks}$ and size $\sim 0.5$ the field of view. It is immediately evident that at lower values of SB the scatter of the simulation outputs is significantly higher. We used these outputs to calculate the systematic uncertainties in our cluster temperature profiles in the following way. We did not calculate the error of a measurement $T_{\mathrm{m}}$ simply as the scatter of the results of the fit of the 200 realizations at temperature $T_{\mathrm{m}}$. Instead we calculated it from the distribution of the "true" temperatures $T_{\mathrm{i}}$ that have a non null probability of being measured as $T_{\mathrm{m}}$. When the SB is high ( $\left.>1 \mathrm{e}-11 \mathrm{erg} \mathrm{s}^{-1} \mathrm{~cm}^{-2} \mathrm{deg}^{-2}\right)$ the systematic scatter of the measurement $T_{\mathrm{i}}$ is very small and the probability that a true temperature $T_{\mathrm{i}} \gg T_{\mathrm{m}}$ would yield a $T_{\mathrm{m}}$ measurement is very low. For example in the case shown in the upper panel of Fig. 11 with a SB $\sim 5 \times 10^{-11} \mathrm{erg} \mathrm{s}^{-1} \mathrm{~cm}^{-2} \mathrm{deg}^{-2}$ the probability that a cluster region with a true temperature $T_{\mathrm{i}}>4$ would be measured $3 \mathrm{keV}$ is almost null. On the contrary, when the $\mathrm{SB}$ is low and the background is comparable or higher than the cluster signal, the scatter of the measurement can be very large, especially at high temperatures. In the middle panel of the same figure, we show the same example for a SB which is a factor 10 lower $\left(5 \times 10^{-11} \mathrm{erg} \mathrm{s}^{-1} \mathrm{~cm}^{-2} \mathrm{deg}^{-2}\right)$ : in this case the probability that $T_{\mathrm{i}}>4$ would be measured $3 \mathrm{keV}$ is not negligible. Thus, to calculate the $T_{\mathrm{m}}$ error, for each $T_{\mathrm{i}}$ of the simulation grid, we calculated the $T_{\mathrm{i}}$ output distribution value in $T_{\mathrm{m}}$; then we calculated the errors as the 16th and 84th percentile of this distribution (lower panel of Fig. 11).

The systematic errors reported in Table 2 and plotted in Fig. 2 are calculated in this way. In most of the spectra of the present sample, the systematic errors do not strongly affect the total error budget of our measurements as the surface brightness of the observed regions is higher than $10^{-11} \mathrm{erg} \mathrm{s}^{-1} \mathrm{~cm}^{-2} \mathrm{deg}^{-2}$. The external regions of the two more distant clusters are the only cases where SB is lower; but here the statistical errors are very large too. However the analysis of the temperature measurement systematic errors is mandatory to realistically calculate the expected errors in the outer regions assuming to have a good statistics data-set as in our simulation of Abell 1795.

Leccardi \& Molendi (2007) shown that, using the most common likelihood estimators, the measurements of the temperatures from outer regions of galaxy clusters are strongly biased. We note that our way of calculating the temperature measurement uncertainties represents a refinement of the usual procedure used in the literature. Indeed, the current approach is a particular case of our procedure, given by the simplifying assumption that the measured value $T_{\mathrm{m}}$ coincide with the true value $T_{\mathrm{i}}$. In the remaining we will refer to this way of calculating the error as $\mathrm{H} 1$, while we will call our refined procedure $\mathrm{H} 2$.

\section{A1795: a case study}

Chandra and XMM-Newton deep observations showed that Abell 1795 is a dynamically relaxed system within $r_{500}$ (Vikhlinin et al. 2006), although a $50 \mathrm{kpc}\left(40^{\prime \prime}\right)$ long X-ray filament in the core is present (Fabian et al. 2001). Thanks to the lower background, Suzaku, for the first time, succeeded in extending the observations in the radial range $r_{500}$ and $r_{200}$ finding a significant asymmetry between the northern and southern part of the cluster. Departure form the hydrostatic equilibrium, with the
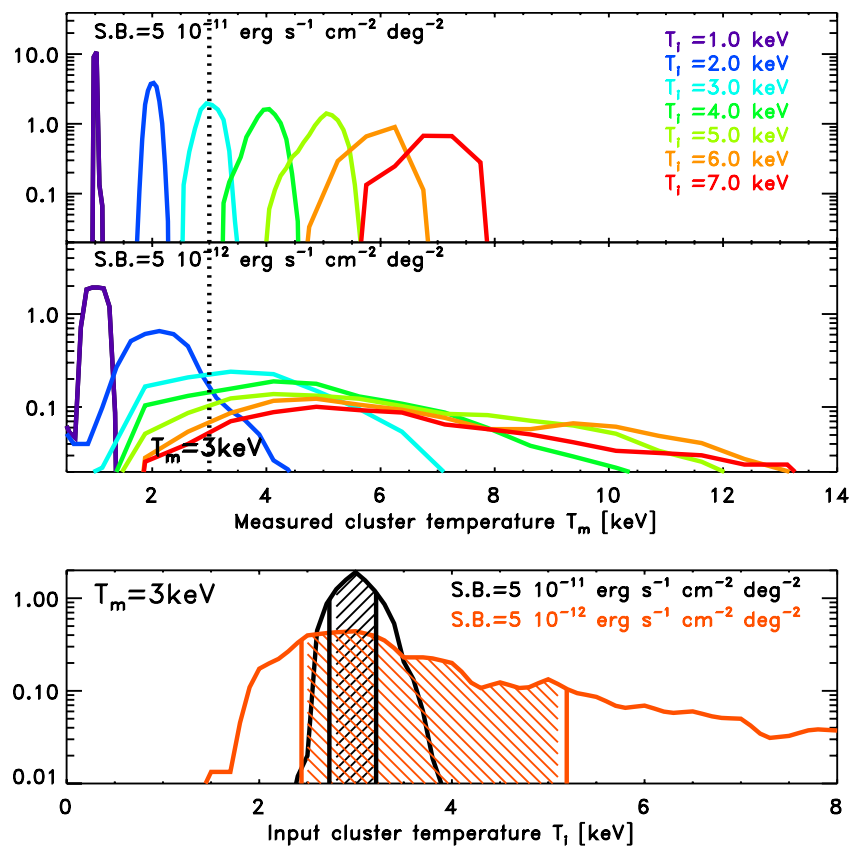

Fig. 11. Upper panel: the simulation outputs relative to an APEC spectrum with $k T=1-7 \mathrm{keV} 100 \mathrm{ks}$ observation extracted from a region size of 0.5 the field of view in a high signal to background ratio regime $\left(S B=5 \times 10^{-11} \mathrm{erg} \mathrm{s}^{-1} \mathrm{~cm}^{-2} \mathrm{deg}^{-2}\right)$. Different colors indicate the distribution of the results of the fits of different input temperatures (200 realizations each). Middle panel: the same of the upper panel in the case of low signal to background ratio $\left(S B=5 \times 10^{-12} \mathrm{erg} \mathrm{s}^{-1} \mathrm{~cm}^{-2} \mathrm{deg}^{-2}\right)$. Lower panel: the distribution of the $T_{\mathrm{i}}$ scatter values measured at $T_{\mathrm{m}}=3 \mathrm{keV}$ relative to the two different values of surface brightness; the intervals between 16th and 84th percentile of both distributions are in evidence.

plasma in-falling from the northern skirt is a possible explanation (Bautz et al. 2009). Suzaku observation sensitivity is limited by two factors: the background from solar wind (SWCX) and the XRB variance (Bautz et al. 2009). The latter was partially mitigated exploiting XMM-Newton or Chandra observations.

In the previous section we described the procedure we developed to quantify the impact of these two factors on Swift XRT temperature measurements for a sample of cluster observed in their central regions. Here we use the same procedures to calculate how accurate a temperature measurement performed by a fairly deep (300 ks) Swift XRT observation of the northern (the brighter) out-skirt of Abell 1795 would be.

We used the (northern) surface brightness and temperature radial profile as measured by Suzaku up to $25^{\prime}$ and reported by (Bautz et al. 2009) to simulate the cluster surface brightness profile for a $300 \mathrm{ks}$ Swift XRT exposure. This was done using a real exposure map in order to account for vignetting, CCD defects and different aim points and roll angles of a real observation. Because the Abell 1795 virial radius largely exceeds the field of view $\left(r_{200}=25.7^{\prime}\right)$, a combination of different pointing is necessary for our purposes. We found that a mosaic of two different aim points with displacement of $13^{\prime}$ from the cluster center optimizes the observation efficiency, providing us with the maximum exposure in the $20^{\prime}-25^{\prime}$ annulus. Starting from $r_{200}$, we calculated the annuli which would contain 1000 events in the $0.5-2.0$ energy band in order to keep the statistical error at the level of $\lesssim 10 \%$.

We used, as XRB, the same of Bautz et al. (2009) which was measured in the most external observed region and is very close and consistent with our median XRB; as NXB the NESR signal 
Table 4. Extraction regions and spectral analysis results of the $300 \mathrm{ks}$ simulated observation.

\begin{tabular}{|c|c|c|c|c|c|c|c|c|c|}
\hline Ann. rad. & $\begin{array}{l}\text { Ann. rad. } \\
{\left[\mathrm{r}_{200}\right]}\end{array}$ & $\begin{array}{c}\text { Area } \\
{\left[\operatorname{deg}^{2}\right]}\end{array}$ & $\begin{array}{l}\text { Exp. } \\
{[\mathrm{ks}]}\end{array}$ & $\begin{array}{c}\text { Surf. Bright. } \\
\text { erg s}^{-1} \mathrm{~cm}^{-2} \mathrm{deg}^{-2} \\
\end{array}$ & Tot. evt & $\begin{array}{c}S /(S+B) \\
{[\%]}\end{array}$ & $\begin{array}{l}T \text { stat. error } \\
{[\text { frac.] }}\end{array}$ & $\begin{array}{c}T \text { syst. error }(\mathrm{H} 1) \\
\text { [frac.] }\end{array}$ & $\begin{array}{c}T \text { syst. error }(\mathrm{H} 2) \\
\text { [frac.] }\end{array}$ \\
\hline $12.6-13.3$ & $0.49-0.52$ & $1.1 \mathrm{E}-02$ & $8.7 \mathrm{E}+01$ & $2.4 \mathrm{E}-11$ & 1504.4 & 69.72 & $0.09-0.10$ & $0.15-0.22$ & $0.15-0.15$ \\
\hline $13.3-14.0$ & $0.52-0.54$ & $1.3 \mathrm{E}-02$ & $8.7 \mathrm{E}+01$ & $2.0 \mathrm{E}-11$ & 1526.7 & 66.05 & $0.09-0.11$ & $0.15-0.22$ & $0.13-0.15$ \\
\hline $14.0-14.9$ & $0.54-0.58$ & $1.6 \mathrm{E}-02$ & $8.6 \mathrm{E}+01$ & $1.6 \mathrm{E}-11$ & 1631.3 & 61.37 & $0.08-0.10$ & .32 & .55 \\
\hline $14.9-16.0$ & $0.58-0.62$ & $1.9 \mathrm{E}-02$ & $9.0 \mathrm{E}+01$ & $1.3 \mathrm{E}-11$ & 1800.0 & 55.64 & $0.09-0.10$ & 0.41 & 0.56 \\
\hline & 68 & $1.9 \mathrm{E}-02$ & $1.1 \mathrm{E}+02$ & & 1909.6 & & & & \\
\hline $17.4-19.0$ & $0.68-0.74$ & $1.9 \mathrm{E}-02$ & $1.2 \mathrm{E}+02$ & $1.1 \mathrm{E}-11$ & 2197.0 & 52.07 & 0.07 & 0.20 & .60 \\
\hline $19.0-20.6$ & $0.74-0.80$ & $1.5 \mathrm{E}-02$ & $1.5 \mathrm{E}+02$ & $9.2 \mathrm{E}-12$ & 2070.5 & 47.39 & $0.07-0.11$ & 0.21 & $0.09-($ \\
\hline $20.6-22.4$ & $80-0.87$ & $1.3 \mathrm{E}-02$ & $1.8 \mathrm{E}+02$ & $9.0 \mathrm{E}-12$ & 2080.4 & 46.78 & 0.12 & 0.21 & $0.10-0.60$ \\
\hline $22.4-25.0$ & $0.87-0.97$ & $1.7 \mathrm{E}-02$ & $1.8 \mathrm{E}+02$ & $6.4 \mathrm{E}-12$ & 2339.5 & 38.39 & $0.09-0.10$ & $0.23-0.39$ & $0.20-1.03$ \\
\hline
\end{tabular}

registered during the observation of GRB 090618 chosen as the most recent observation lasting more than $300 \mathrm{ks}$,

Thus, for each annulus we calculate the total (statistical + systematics) error in temperature measurement using the recipes described in Sect. 4.

Results of this procedure are reported in Table 4 and shown in the upper panel of Fig. 12 in comparison with the Suzaku measurement. In the table, for each annulus we report the radii, the area, the effective exposure time (accounting for exposure maps and vignetting), the expected mean cluster surface brightness, the expected total events and the source/total ratio and the expected errors. We report explicitly the relative contribution of the statistics and the systematics terms. The latter are calculated either in the assumption that Suzaku measurement is the true value (H1) either considering the distribution of the possible true temperature values as expected from our simulation (H2) as explained in Sect. 4. The errors are reported at $90 \%$ of confidence consistently with Suzaku published numbers (Bautz et al. 2009).

Systematic errors were calculated, first, assuming the Suzaku measurement as the true value of the temperature. This was done following the approach of Bautz et al. (2009) to compare the Swift XRT expected accuracy with Suzaku (H1 column in Table 4 and black error bars in Fig. 12). The simulated spectrum from the external bin is shown in Fig. 13. We found that a $300 \mathrm{ks}$ Swift-XRT observation would significantly improve the accuracy of the Suzaku temperature measurement in the (northern) outskirts of the cluster Abell 1795, both in terms of spatial binning and relative accuracy. Indeed in the annulus within 17-25' while Suzaku could measure only one single temperature with an accuracy of $\sim 60 \%$, we expect that the XRT observation would be able to measure four different temperatures with an accuracy of $\sim 40 \%$.

Second, we refined the error calculation, also considering the distribution of the possible true temperatures which could yield the measured value (H2 procedure, see Sect. 4). In this case the upper error bars are significantly larger. This is the result of the not negligible bias in the high temperature $(T>5 \mathrm{keV})$ measurement in the low SB regime (Sect. 4 and Fig. 11). In this case in the three bins within 17-23' we expect an accuracy of $\sim 60 \%$ similarly to the Suzaku one, while in the last bin $\left(23-25^{\prime}\right)$ the upper error bar is $\sim 100 \%$ of the measured value ( $\mathrm{H} 2$ column in Table 4 and red error bars in Fig. 12). We note that the impact of the bias in the high temperature $(T>5 \mathrm{keV})$ measurement in a low SB regime on temperature accuracy, has never been quantified for Suzaku. Even in the unrealistic assumption that this is completely negligible, the Swift XRT observation would improve the accuracy of the temperature profile significantly narrowing the spatial binning up to $\sim R_{200}$.

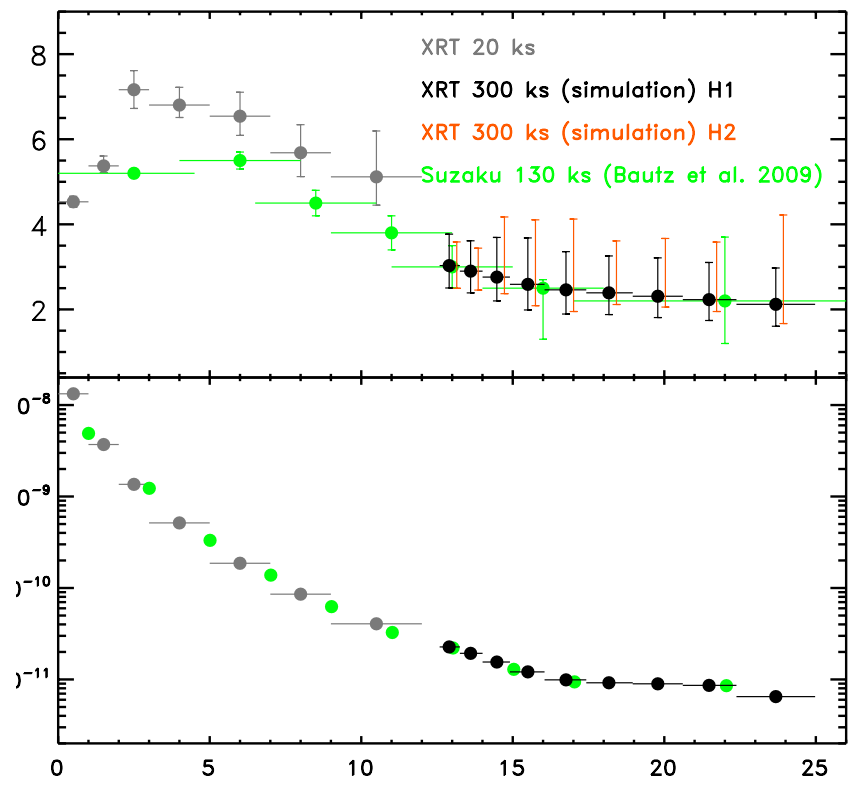

Fig. 12. Simulation of 300 ks observation of the northern skirt of Abell 1795. Upper panel: surface brightness of the cluster. Green points are from Suzaku observation (Bautz et al. 2009). Grey points are from Swift XRT observation (already shown in Fig. 2). Black points would be the result of $300 \mathrm{ks}$ observation. Lower panel: the surface brightness profile of the cluster as observed by Suzaku, with the same color code. XRT spatial bins in the simulation are chosen in order to have a minimum of 1000 source counts in the $0.7-2.0 \mathrm{keV}$ band.

In a spatially resolved spectral study of a low surface brightness source, a telescope reaches the limit of its capabilities, when a deep observation allows to map the source at the best of its angular resolution (bins $\geq 3$ times the HEW, to avoid PSF mixing) and, at the same time, in each single bin, the collected photons are enough to make the statistic error negligible with respect to the systematics (mainly the background ones). The Suzaku telescope, in its observation of Abell 1795, almost reached its limits: in fact, most of the error in the temperature measurement, is due to background variance, while the angular resolution can be only slightly improved as the telescope HEW is $2^{\prime}$. On the other hand, we showed that, with a $300 \mathrm{ks}$ observation, Swift-XRT could reach the same level of accuracy with an angular resolution of $\sim 2$. This leaves a wide range of improvement: indeed, doubling the observation exposure would allow to halve the spatial binning, as the HEW is $18^{\prime \prime}$. 


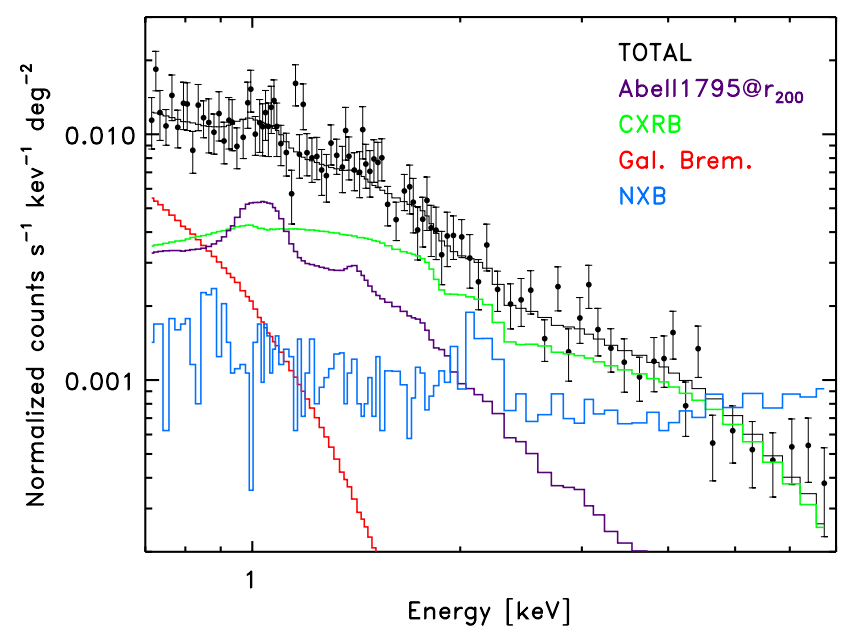

Fig. 13. Simulation of the $300 \mathrm{ks}$ of the last annulus.

\section{Discussion and conclusion}

In the outer regions of nearby clusters, the ICM emission is only a small fraction of the whole signal collected by the detector. The regime, where the background systematics, affect the spectroscopic measurements much more than the statistical error, is easily reached. While XMM-Newton and Chandra are not suitable for this kind of observation due to the high level of particle background, in all the published works presenting Suzaku observations of cluster outskirts the evaluation of the XRB and its variance is the main issue. Different approaches have been pursued. Bautz et al. (2009) and Hoshino et al. (2010), in their studies of Abell 1795 and Abell 1413 respectively, used the signal from external regions at $\sim 1.2 \times r_{200}$ as XRB, in the assumption that the cluster emission is negligible at that distance from the center. To study the temperature profile of PKS0745-19, George et al. (2009) used the Lockman Hole observation which was performed just few days before the cluster observation. Interestingly they found significant emission from the cluster ICM at distance $>1.5 \times r_{200}$. Kawaharada et al. (2010) used the two closest observations (at $\sim 8^{\circ}$ of distance) among the ones suitable as blank fields in the Suzaku archive. Reiprich et al. (2009) and Fujita et al. (2008) used the classical models from literature to fit the cluster spectrum together with the XRB (using a different choice of free parameters).

In this paper we presented for the first time the analysis of the Swift-XRT observations of a sample of 6 galaxy clusters. We measured the temperature profiles as far as $\sim 0.5 R_{200}$ for all (but Coma) sample clusters. To estimate the cosmic background we used a statistical approach, modeling a representative sample of blank fields, with a sky-coverage of $\sim 15 \mathrm{deg}^{2}$. We used the blank field median spectrum as XRB model to fit our cluster spectra. We calculated the systematics of this approach by simulating realistic clusters with different temperatures and surface brightnesses summed to real XRB data extracted from blank fields with different sizes and exposure times. With this approach in the systematics calculation, exploiting a statistically fair sample of BF, we directly accounted for the XRB variance (for both CXRB and GXRB). Moreover we presented a new way to calculate the uncertainties in the temperature measurements significantly refining the current approach in literature. Given a measure $T_{\mathrm{m}}$, we accounted for the possibility that $T_{\mathrm{m}}$ is produced by a $T_{\mathrm{i}} \neq T_{\mathrm{m}}$. This allowed us to realistically simulate the temperature measurement in the outer regions of Abell 1795 which would be provided by a deep XRT observation. We showed that, thanks to an unprecedented combination of low background, good PSF the Swift XRT would be able to significantly improve the current accuracy of the temperature measurements in the outer regions of nearby clusters.

The ideal telescope for cluster outskirts observation would be a large grasp (wide field and large collecting area) and low background telescope such the proposed WFXT (Murray et al. 2010). In the next decade eRosita will be the only mission operative with these characteristics (Predehl et al. 2010), a grasp 10 times larger than XMM (100 times larger than Swift-XRT). Interestingly, eRosita, with an effective area of $\sim 1500 \mathrm{~cm}^{2}$ at $1.5 \mathrm{keV}(\sim 10$ times larger than XRT) and an expected background of $\sim 9$ counts $\mathrm{s}^{-1} \mathrm{deg}^{-2}(\sim 10$ times larger than XRT) will have the same source/background ratio of the Swift-XRT when observing extended sources. If these numbers will be confirmed in flight, and the NXB will be reproduced with the same accuracy $(\$ 3 \%)$, at a given value of surface brightness, eRosita will have systematic errors on temperature measurements which will be very close to the ones we found for Swift-XRT. In this case, our proposed XRT observations would represent a pilot for the eRosita mission; on the other hand, if the NXB of eRosita will be higher than expected (eRosita will be the first X-ray telescope positioned in L2) or it will be impossible to reproduce it with the same accuracy, the XRT observation would remain the only way to improve our knowledge of the cluster outskirts physics at least for the next decade.

Acknowledgements. We acknowledge the financial contribution from contracts ASI-INAF I/023/05/0, I/088/06/0, I/011/07/0, I/009/10/0.

This research has made use of:

- the X-Rays Clusters Database (BAX) which is operated by the Laboratoire d'Astrophysique de Tarbes-Toulouse (LATT), under contract with the Centre National d'Etudes Spatiales (CNES);

- the NASA's Astrophysics Data System;

- the NASA/IPAC Extragalactic Database (NED) which is operated by the Jet Propulsion Laboratory, California Institute of Technology, under contract with the National Aeronautics and Space Administration.

\section{References}

Allen, S. W., Schmidt, R. W., \& Fabian, A. C. 2001, MNRAS, 328, L37 Arnaud, M., Aghanim, N., Gastaud, R., et al. 2001, A\&A, 365, L67 Arnaud, M., Pointecouteau, E., \& Pratt, G. W. 2005, A\&A, 441, 893 Arnaud, M., Pratt, G. W., Piffaretti, R., et al. 2010, A\&A, 517, A92 Bautz, M. W., Miller, E. D., Sanders, J. S., et al. 2009, PASJ, 61, 1117 Burrows, D. N., Hill, J. E., Nousek, J. A., et al. 2005, Space Sci. Rev., 120, 165 Citterio, O., Conconi, P., Ghigo, M., et al. 1994, in SPIE Conf. Ser., ed. R. B. Hoover, \& A. B. Walker, 2279, 480

De Grandi, S., \& Molendi, S. 2002, ApJ, 567, 163

Ettori, S., \& Balestra, I. 2009, A\&A, 496, 343

Ettori, S., \& Molendi, S. 2010, [arXiv: 1005.0382]

Fabian, A. C., Sanders, J. S., Ettori, S., et al. 2001, MNRAS, 321, L33

Fujita, Y., Tawa, N., Hayashida, K., et al. 2008, PASJ, 60, 343

Gehrels, N., Chincarini, G., Giommi, P., et al. 2004, ApJ, 611, 1005

George, M. R., Fabian, A. C., Sanders, J. S., Young, A. J., \& Russell, H. R. 2009, MNRAS, 395, 657

Godet, O., Beardmore, A. P., Abbey, A. F., et al. 2009, A\&A, 494, 775 Hoshino, A., Patrick Henry, J., Sato, K., et al. 2010, PASJ, 62, 371

Kalberla, P. M. W., Burton, W. B., Hartmann, D., et al. 2005, A\&A, 440, 775

Kawaharada, M., Okabe, N., Umetsu, K., et al. 2010, ApJ, 714, 423

Koester, B. P., McKay, T. A., Annis, J., et al. 2007, ApJ, 660, 239

Kuntz, K. D., \& Snowden, S. L. 2000, ApJ, 543, 195

Kushino, A., Ishisaki, Y., Morita, U., et al. 2002, PASJ, 54, 327

Leccardi, A., \& Molendi, S. 2007, A\&A, 472, 21

Leccardi, A., \& Molendi, S. 2008, A\&A, 486, 359

Moretti, A., Campana, S., Lazzati, D., \& Tagliaferri, G. 2003, ApJ, 588, 696

Moretti, A., Campana, S., Mineo, T., et al. 2005, in SPIE Conf. Ser., ed. O. H. W. Siegmund, 5898, 360 
Moretti, A., Campana, S., Tagliaferri, G., et al. 2004, in SPIE Conf. Ser., ed. K. A. Flanagan, \& O. H. W. Siegmund, 5165, 232

Moretti, A., Pagani, C., Cusumano, G., et al. 2009, A\&A, 493, 50

Moretti, A., Perri, M., Capalbi, M., et al. 2007, in SPIE Conf. Ser., ed. S. L. O’Dell, \& G. Pareschi, 6688

Murray, S. S., Giacconi, R., Ptak, A., et al. 2010, in SPIE Conf. Ser., 7732

Neumann, D. M. 2005, A\&A, 439, 465

Owen, F. N., Ledlow, M. J., \& Keel, W. C. 1995, AJ, 109, 14

Pointecouteau, E., Arnaud, M., \& Pratt, G. W. 2005, A\&A, 435, 1

Predehl, P., Andritschke, R., Böhringer, H., et al. 2010, in SPIE Conf. Ser., 7732

Rasheed, B., Bahcall, N., \& Bode, P. 2010, PNAS, submitted

[arXiv: 1007.1980$]$
Reiprich, T. H., Hudson, D. S., Zhang, Y., et al. 2009, A\&A, 501, 899 Revnivtsev, M., Molkov, S., \& Sazonov, S. 2008, A\&A, 483, 425

Roncarelli, M., Ettori, S., Dolag, K., et al. 2006, MNRAS, 373, 1339

Snowden, S. L., Egger, R., Finkbeiner, D. P., Freyberg, M. J., \& Plucinsky, P. P. 1998, ApJ, 493, 715

Snowden, S. L., Mushotzky, R. F., Kuntz, K. D., \& Davis, D. S. 2008, A\&A, 478,615

Vikhlinin, A., Forman, W., \& Jones, C. 1999, ApJ, 525, 47

Vikhlinin, A., Markevitch, M., Murray, S. S., et al. 2005, ApJ, 628, 655

Vikhlinin, A., Kravtsov, A., Forman, W., et al. 2006, ApJ, 640, 691

Wen, Z. L., Han, J. L., \& Liu, F. S. 2009, ApJS, 183, 197 